\title{
Automorphic forms on the 5-dimensional complex ball with respect to the Picard modular group over $\mathbb{Z}[i]$
}

\author{
K. Matsumoto, T. Minowa and R. Nishimura
}

(Received September 2, 2005)

\begin{abstract}
We represent the 105 automorphic forms on the 5-dimensional complex ball $\mathbb{B}^{5}$ constructed by Matsumoto-Terasoma as the products of four linear combinations of the pull backs of theta constants under an embedding of $\mathbb{B}^{5}$ into the Siegel upper half space of degree 6. They were used to describe the inverse of the period map for the family of the 4-fold coverings of the complex projective line branching at eight points.
\end{abstract}

Key words: automorphic forms, theta constants.

\section{Introduction}

The period map for the family of the 4-fold coverings of the complex projective line $\mathbb{P}^{1}$ branching at eight points is studied in [MT]. Its inverse is given by 105 automorphic forms $f_{J}$ on the 5 -dimensional complex ball $\mathbb{B}^{5}$ with respect to the monodromy group of the period map, where $J$ are $(2,2,2,2)$-partitions $\left\{j_{1} j_{2} ; j_{3} j_{4} ; j_{5} j_{6} ; j_{7} j_{8}\right\}$ of $\{1, \ldots, 8\}$. The automorphic form $f_{J_{1}}$ for $J_{1}=\langle 12 ; 34 ; 56 ; 78\rangle$ is given by the pull back of the product of four theta constants under the embedding $\imath$ of $\mathbb{B}^{5}$ into the Siegel upper half space $\mathbb{S}^{6}$ of degree 6 induced by the period map. The symmetric group $S_{8}$ of degree 8 transitively acts on the set of $(2,2,2,2)$-partitions, and it is represented in the symplectic group $S p_{6}(\mathbb{Q})$ (not in $S p_{6}(\mathbb{Z})$ ) by the period map. The group $S_{8}$ represented in $S p_{6}(\mathbb{Q})$ acts on $f_{J_{1}}$ as the transformation of theta constants on $\mathbb{S}^{6}$, and this action yields all $f_{J}$ from $f_{J_{1}}$. Actually, few $f_{J}$ are explicitly given in terms of theta constants in [MT] since transformation formulas of theta constants for some elements of $S p_{6}(\mathbb{Q})$ were not known.

In this paper, we represent all $f_{J}$ as the products of four linear combinations of the pull backs of theta constants under the embedding $\imath: \mathbb{B}^{5} \rightarrow$ $\mathbb{S}^{6}$. The set of $(2,2,2,2)$-partitions can be regarded as the quotient $G \backslash S_{8}$, where $G$ is the isotropy subgroup of $S_{8}$ for the partition $J_{1}$. The group

2000 Mathematics Subject Classification : 11F55, 32N15, 14J15. 
$G$ is represented in $S p_{6}(\mathbb{Z})$ by the period map, and the double quotient $G \backslash S_{8} / G$ consists of five elements: the unit $\sigma_{1}, \sigma_{2}=(23), \sigma_{3}=(23)(45)$, $\sigma_{4}=(23)(67)$ and $\sigma_{5}=(23)(45)(67)$, where $(j k)$ is the transposition of $j$ and $k$. We give transformation formulas of the theta constants for the elements of $S p_{6}(\mathbb{Q})$ corresponding to $(23)$, (45) and $(67)$, which yield the explicit forms $f_{J_{j}}$ of the actions of $\sigma_{j}$ on $f_{J_{1}}$. We can obtain all $f_{J}$ by acting $G$ represented in $S p_{6}(\mathbb{Z})$ on $f_{J_{2}}, \ldots, f_{J_{5}}$. We have to study the constant $\kappa(\sigma)$ in the transformation formula of the theta constants in [I] for the elements in $S p_{6}(\mathbb{Z})$ corresponding to generators of $G$ in order to give linear relations among $f_{J}$. Some linear relations among $f_{J}$ yield quadratic relations among pull backs of theta constants under $\imath$.

Another expression of fourteen linearly independent $f_{J}$ in terms of liftings is given in $[\mathrm{K}]$.

\section{Automorphic forms on $\mathbb{B}^{5}$}

\subsection{5 polynomials}

Let $J$ be a $(2,2,2,2)$-partition of the set $\{1, \ldots, 8\}$ :

$$
\begin{aligned}
J & =\left\langle j_{1} j_{2} ; j_{3} j_{4} ; j_{5} j_{6} ; j_{7} j_{8}\right\rangle, \\
\left\{j_{1}, \ldots, j_{8}\right\} & =\{1, \ldots, 8\}, \quad j_{1}<j_{2}, j_{3}<j_{4}, j_{5}<j_{6}, j_{7}<j_{8} .
\end{aligned}
$$

The cardinality of the set $\mathcal{J}$ of $(2,2,2,2)$-partitions of $\{1, \ldots, 8\}$ is

$$
\left(\begin{array}{l}
8 \\
2
\end{array}\right)\left(\begin{array}{l}
6 \\
2
\end{array}\right)\left(\begin{array}{l}
4 \\
2
\end{array}\right) / 4 !=\frac{8 !}{2^{4} \times 4 !}=105 .
$$

For each element of $J \in \mathcal{J}$, we define a polynomial

$$
x_{J}=\left(x_{j_{1}}-x_{j_{2}}\right)\left(x_{j_{3}}-x_{j_{4}}\right)\left(x_{j_{5}}-x_{j_{6}}\right)\left(x_{j_{7}}-x_{j_{8}}\right)
$$

of variables $x_{1}, \ldots, x_{8}$.

The symmetric group $S_{8}$ acts on $x={ }^{t}\left(x_{1}, \ldots, x_{8}\right) \in \mathbb{C}^{8}$ from the left as the transposition of coordinates, which can be represented as the left multiple $\sigma \cdot x$, where $\sigma \in S_{8}$ and we regard $S_{8}$ as naturally embedded in $G L_{8}(\mathbb{Z})$. Note that

$$
\begin{aligned}
(123) \cdot x & =((12)(23)) \cdot x=(12) \cdot{ }^{t}\left(x_{1}, x_{3}, x_{2}, \ldots\right)={ }^{t}\left(x_{3}, x_{1}, x_{2}, \ldots\right), \\
(132) \cdot x & =((23)(12)) \cdot x=(23) \cdot{ }^{t}\left(x_{2}, x_{1}, x_{3}, \ldots\right)={ }^{t}\left(x_{2}, x_{3}, x_{1}, \ldots\right),
\end{aligned}
$$

where $\left(j_{1}, \ldots, j_{k}\right)$ denotes a cyclic permutation. If we regard $\sigma \in S_{8}$ as a 
map $\sigma:\{1, \ldots, 8\} \ni k \mapsto \sigma(k) \in\{1, \ldots, 8\}$, this action is equivalent to

$$
\sigma \cdot{ }^{t}\left(x_{1}, \ldots, x_{8}\right)={ }^{t}\left(x_{\sigma^{-1}(1)}, \ldots, x_{\sigma^{-1}(8)}\right) .
$$

The group $S_{8}$ acts on $x_{J}$ from the right as its pull-back under $\sigma \in S_{8}$, i.e., $x_{J}^{\sigma}=\sigma^{*}\left(x_{J}\right)$. Note that $x_{J}^{\sigma \sigma^{\prime}}=\left(\sigma^{\prime}\right)^{*}\left(x_{J}^{\sigma}\right)$. For examples,

$$
\begin{aligned}
& x_{J_{1}}^{(12)(23)}=\left(x_{3}-x_{1}\right)\left(x_{2}-x_{4}\right)\left(x_{5}-x_{6}\right)\left(x_{7}-x_{8}\right)=-x_{\langle 13 ; 24 ; 56 ; 78\rangle}, \\
& x_{J_{1}}^{(23)(12)}=\left(x_{2}-x_{3}\right)\left(x_{1}-x_{4}\right)\left(x_{5}-x_{6}\right)\left(x_{7}-x_{8}\right)=x_{\langle 23 ; 14 ; 56 ; 78\rangle},
\end{aligned}
$$

where $J_{1}$ is the $(2,2,2,2)$-partition $\langle 12 ; 34 ; 56 ; 78\rangle$. We define a subgroup $G$ of $S_{8}$ as

$$
G=\left\{\sigma \in S_{8} \mid x_{J_{1}}^{\sigma}= \pm x_{J_{1}}\right\},
$$

which is generated by

$$
\text { (12), (34), (56), (78), (13)(24), (35)(46), (57)(68). }
$$

Note that $G$ is a maximal subgroup of order $2^{4} \cdot 4$ ! and that the set $\mathcal{J}$ can be regarded as the quotient $G \backslash S_{8}$.

\subsection{Theta constants}

Let $\mathbb{S}^{r}$ be the Siegel upper half space of degree $r$, which is the set of symmetric $r \times r$ complex matrices whose imaginary parts are positive definite. The symplectic group

$$
S p_{r}(\mathbb{Q})=\left\{M \in S L_{2 r}(\mathbb{Q}) \mid{ }^{t} M\left(\begin{array}{ll}
{ }^{t} & -I_{r} \\
I_{r} &
\end{array}\right) M=\left(\begin{array}{ll}
I_{r} & -I_{r}
\end{array}\right)\right\}
$$

acts on $\mathbb{S}^{r}$ as

$$
M \cdot \tau=(A \tau+B)(C \tau+D)^{-1}, \quad \tau \in \mathbb{S}_{r}, M=\left(\begin{array}{ll}
A & B \\
C & D
\end{array}\right) \in S p_{r}(\mathbb{Q}),
$$

where $I_{r}$ denotes the unit matrix of degree $r$.

Theta constants with half characteristics $a, b \in \mathbb{Z}^{r}$ on $\mathbb{S}^{r}$ are defined as

$$
\vartheta_{a, b}(\tau)=\sum_{n \in \mathbb{Z}^{r}} \exp \left(\pi i\left(n+\frac{a}{2}\right) \tau^{t}\left(n+\frac{a}{2}\right)+2 \pi i\left(n+\frac{a}{2}\right) \frac{{ }^{t} b}{2}\right),
$$

$\tau \in \mathbb{S}^{r}$. 
They satisfy

$$
\vartheta_{a+2 p, b+2 q}(\tau)=\exp \left(\pi i a^{t} q\right) \vartheta_{a, b}(\tau)
$$

for $p, q \in \mathbb{Z}^{r}$. The following transformation formula for theta constants by the action of $S p_{r}(\mathbb{Z})=S p_{r}(\mathbb{Q}) \cap S L_{2 r}(\mathbb{Z})$ is a basic material of this paper.

Fact 1 (Corollary in p. $176[\mathrm{I}]$ ) $\quad$ For $M=\left(\begin{array}{ll}A & B \\ C & D\end{array}\right) \in S p_{r}(\mathbb{Z})$, put

$$
\begin{aligned}
M \cdot(a, b)= & (a, b) M^{-1}+\left(\left(C^{t} D\right)_{0},\left(A^{t} B\right)_{0}\right), \\
\phi_{a, b}(M)= & -\frac{1}{8}\left(a^{t} D B^{t} a-2 a^{t} B C^{t} b+b^{t} C A^{t} b\right) \\
& +\frac{1}{4}\left(a^{t} D-b^{t} C\right)^{t}\left(A^{t} B\right)_{0},
\end{aligned}
$$

where $A_{0}$ is the row vector consisting of the diagonal components of a square matrix $A$. We have

$$
\vartheta_{M \cdot(a, b)}(M \cdot \tau)=\kappa(M) \exp \left(2 \pi i \phi_{a, b}(M)\right) \sqrt{\operatorname{det}(C \tau+D)} \vartheta_{a, b}(\tau),
$$

where $\kappa(M)^{2}$ is \pm 1 or $\pm i$ depending only on $M$.

\subsection{An embedding of $\mathbb{B}^{5}$ into $\mathbb{S}^{6}$}

We put $\mathbb{B}^{5}=\left\{y \in \mathbb{P}^{5} \mid y^{*} H^{-1} y<0\right\}$, where

$$
H=\left(\begin{array}{cccccc}
2 & -1+i & & & & \\
-1-i & 2 & -1+i & & & \\
& -1-i & 2 & -1+i & & \\
& & -1-i & 2 & -1+i & \\
& & & -1-i & 2 & -1+i \\
& & & & -1-i & 2
\end{array}\right)
$$

Since the signature of $H$ is $(5,1), \mathbb{B}^{5}$ is isomorphic to the 5-dimensional complex ball $\left\{\left.\left(z_{1}, \ldots, z_{5}\right) \in \mathbb{C}^{5}|| z_{1}\right|^{2}+\cdots+\left|z_{5}\right|^{2}<1\right\}$. We define discrete subgroups acting on $\mathbb{B}^{5}$ as follows:

$$
\begin{aligned}
\Gamma & =\left\{g \in G L_{6}(\mathbb{Z}[i]) \mid g^{*} H^{-1} g=H^{-1}\right\} \\
& =\left\{g \in G L_{6}(\mathbb{Z}[i]) \mid g H g^{*}=H\right\}, \\
\Gamma(1-i) & =\left\{g \in \Gamma \mid g \equiv I_{6} \bmod (1-i)\right\} .
\end{aligned}
$$


It is shown in [MY] that the quotient group $\Gamma / \Gamma(1-i)$ is isomorphic to $S_{8}$ and that $\Gamma$ is generated by seven unitary reflections of order four:

$$
g_{j}=I_{6}-(1-i) H v_{j}^{*} v_{j} /\left(v_{j} H v_{j}^{*}\right)
$$

where

$$
\begin{array}{ll}
v_{1}=(1,0,0,0,0,0), & v_{2}=(0,1,0,0,0,0), \\
v_{3}=(0,0,1,0,0,0), & v_{4}=(0,0,0,1,0,0), \\
v_{5}=(0,0,0,0,1,0), & v_{6}=(0,0,0,0,0,1), \\
v_{7}=(1,1-i,-i, 0,1,1-i) .
\end{array}
$$

The reflections $g_{j}$ satisfy $g_{j}^{2} \in \Gamma(1-i)$ and the braid relations $g_{j} g_{j+1} g_{j}=$ $g_{j+1} g_{j} g_{j+1}$. We put $g_{j, j+1}=g_{j}$ and

$$
g_{j k}=\left(g_{k-1} \cdots g_{j+2} g_{j+1}\right) g_{j}\left(g_{k-1} \cdots g_{j+2} g_{j+1}\right)^{-1}
$$

for $k>j+1$. There exists a homomorphism $s: \Gamma \rightarrow S_{8}$ such that $s\left(g_{j k}\right)$ is the transposition $(j, k)$.

The domain $\mathbb{B}^{5}$ is embedded into the Siegel upper half space $\mathbb{S}^{6}$ of degree 6 as follows:

$$
\imath: \mathbb{B}^{5} \ni y \mapsto \tau=i\left[U-2 \frac{(T y)^{t}(T y)}{t(T y) U(T y)}\right] \in \mathbb{S}^{6},
$$

where $T=N_{1}-i N_{2}$,

$$
\begin{aligned}
& N_{1}=\left(\begin{array}{cccccc}
1 & 0 & 0 & 0 & 0 & 0 \\
1 & 1 & 0 & 0 & 0 & 0 \\
1 & 1 & 0 & 0 & 0 & 0 \\
1 & 1 & 0 & -1 & 0 & 0 \\
1 & 1 & 0 & 0 & 1 & 0 \\
1 & 1 & 0 & 0 & 1 & 1
\end{array}\right), \quad N_{2}=\left(\begin{array}{cccccc}
0 & 0 & 0 & 0 & 0 & 0 \\
0 & 1 & 0 & 0 & 0 & 0 \\
0 & 1 & 1 & 0 & 0 & 0 \\
0 & 1 & 1 & 1 & 0 & 0 \\
0 & 1 & 1 & 0 & 0 & 0 \\
0 & 1 & 1 & 0 & 0 & 1
\end{array}\right), \\
& U=\left(\begin{array}{llll}
I_{2} & & & \\
& & -1 & \\
& -1 & & \\
& & & I_{2}
\end{array}\right) \text {. }
\end{aligned}
$$

This embedding $\imath$ induces a homomorphism

$$
\jmath: \Gamma \ni g \mapsto N\left(\begin{array}{cc}
\operatorname{Re}(g) & -\operatorname{Im}(g) \\
\operatorname{Im}(g) & \operatorname{Re}(g)
\end{array}\right) N^{-1} \in S p_{6}(\mathbb{Q}),
$$


where $N=\left(\begin{array}{cc}N_{1} & N_{2} \\ U N_{2} & -U N_{1}\end{array}\right)$. Note that

$$
\jmath(g)=\left(\begin{array}{cc}
A & B \\
-U B U & U A U
\end{array}\right),
$$

where $6 \times 6$ real matrices $A, B$ are given by $T g T^{-1}=A+i B U$, and that $\left(T g T^{-1}\right) U\left(T g T^{-1}\right)^{*}=U$.

The maps $\imath$ and $\jmath$ satisfy

$$
\imath(g y)=(A \imath(y)+B)(C \imath(y)+D)^{-1}
$$

for $g \in \Gamma$ and $\jmath(g)=\left(\begin{array}{ll}A & B \\ C & D\end{array}\right) \in S p_{6}(\mathbb{Q})$.

We put $\Psi(M, \tau)=\operatorname{det}(C \tau+D)$ for $M=\left(\begin{array}{ll}A & B \\ C & D\end{array}\right) \in S p_{6}(\mathbb{Q})$ and $\tau \in \mathbb{S}^{6}$.

Lemma 1 For $y \in \mathbb{B}^{5}, g \in \Gamma$ and $\jmath(g)=\left(\begin{array}{ll}A & B \\ C & D\end{array}\right) \in S p_{6}(\mathbb{Q})$, we have

$$
\Psi(\jmath(g), \imath(y))=\operatorname{det}(C \imath(y)+D)=\frac{{ }^{t}(T g y) U(T g y)}{\operatorname{det}(g)^{t}(T y) U(T y)} .
$$

Proof. We have

$$
\operatorname{det}(C \imath(y)+D)=\operatorname{det}(U(-B U \tau U+A) U)=\operatorname{det}(-B U \tau U+A)
$$

where $\tau=\imath(y), g \in \Gamma$ and $T g T^{-1}=A+i B U$. We put $X=-B U \tau U+A$, and $z=T y$. Since

$$
\tau U=i\left[I_{6}-2 \frac{z^{t} z U}{t_{z U z}}\right]
$$

we have $\tau U z=-i z$, and $\tau U w_{j}=i w_{j}$, where $w_{1}, \ldots, w_{5}$ span the subspace $W=\left\{w \in \mathbb{C}^{6} \mid{ }^{t} z U w=0\right\}$. Thus

$$
\begin{aligned}
X z & =-B U(-i z)+A z=T g T^{-1} z, \\
X w_{j} & =-B U\left(i w_{j}\right)+A w_{j}=\overline{T g T^{-1}} w_{j}(j=1, \ldots, 5) .
\end{aligned}
$$

We express $X z$ as the linear combination of $\overline{T g T^{-1}} z$ and $\overline{T g T^{-1}} w_{j}(j=$ 
$1, \ldots, 5):$

$$
X z=c \overline{T g T^{-1}} z+\sum_{j=1}^{5} c_{j} \overline{T g T^{-1}} w_{j}
$$

Note that $\operatorname{det}(X)$ is equal to $c \operatorname{det}\left(\overline{T g T^{-1}}\right)=c \operatorname{det}(\bar{g})=c / \operatorname{det}(g)$. Multiply ${ }^{t} z U\left(\overline{T g T^{-1}}\right)^{-1}$ from the left of this linear combination, then we have

$$
{ }^{t} z U\left(\overline{T g T^{-1}}\right)^{-1} X z=c^{t} z U z
$$

since ${ }^{t} z U w_{j}=0$. The left hand side of this equality is

$$
\begin{aligned}
{ }_{z}^{t} U\left(\overline{\operatorname{TgT}^{-1}}\right)^{-1}\left(T g T^{-1}\right) z & ={ }^{t} z U\left(U^{t}\left(T g T^{-1}\right) U\right)\left(T g T^{-1}\right) z \\
& ={ }^{t}(T g y) U(T g y),
\end{aligned}
$$

since $\left(T g T^{-1}\right)^{*} U\left(T g T^{-1}\right)=U$.

We put

$$
M_{j}=\left(\begin{array}{ll}
A_{j} & B_{j} \\
C_{j} & D_{j}
\end{array}\right)=\left(\begin{array}{cc}
A_{j} & B_{j} \\
-U B_{j} U & U A_{j} U
\end{array}\right)=\jmath\left(g_{j}\right),
$$

for $j=1, \ldots, 7$, and $M_{j k}=\jmath\left(g_{j k}\right)$ for $1 \leq j<k \leq 8$.

Fact 2 The $(1,1)$-block $A_{j}$ and the $(1,2)$-block $B_{j}$ of $M_{j}=\jmath\left(g_{j}\right)$ are given as follows:

$$
\begin{aligned}
& A_{1}=\left(\begin{array}{ll}
0 & \\
& I_{5}
\end{array}\right), \quad B_{2}=\left(\begin{array}{ll}
1 & \\
& O_{5}
\end{array}\right), \\
& A_{2}=\left(\begin{array}{lll}
I_{2}-\Delta & \\
& & I_{4}
\end{array}\right), \quad B_{2}=\left(\begin{array}{ll}
\Delta & \\
& O_{4}
\end{array}\right), \\
& A_{3}=\left(\begin{array}{lllll}
1 & & & & \\
& 0 & 1 & & \\
& & 1 & & \\
& -1 & 1 & 1 & \\
& & & 1 & \\
& & & & 1
\end{array}\right), \quad B_{3}=\left(\begin{array}{ccccc}
0 & & & \\
& 1 & 1 & \\
& & 0 & & \\
& 1 & 1 & \\
& & & 0 & \\
& & & & 0
\end{array}\right) \\
& A_{4}=\left(\begin{array}{llll}
I_{2} & & & \\
& I_{2}-\Delta & \\
& & I_{2}
\end{array}\right), \quad B_{4}=\left(\begin{array}{lll}
O_{2} & & \\
& \Delta & \\
& & O_{2}
\end{array}\right),
\end{aligned}
$$




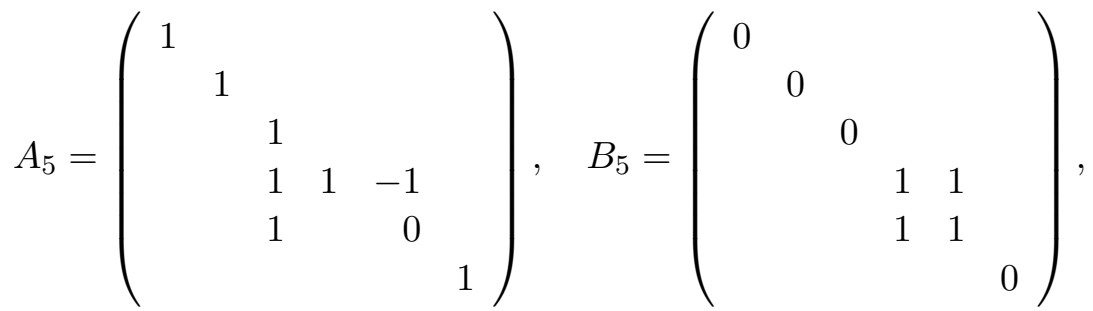

$$
\begin{aligned}
& A_{6}=\left(\begin{array}{ll}
I_{4} & \\
& I_{2}-\Delta
\end{array}\right), \quad B_{6}=\left(\begin{array}{ll}
O_{4} & \\
& \Delta
\end{array}\right) \text {, } \\
& A_{7}=\left(\begin{array}{cc}
I_{5} & \\
& 0
\end{array}\right), \quad B_{7}=\left(\begin{array}{ll}
O_{5} & \\
& 1
\end{array}\right) \text {, }
\end{aligned}
$$

where $O_{k}$ is the zero matrix of degree $k$ and

$$
\Delta=\frac{1}{2}\left(\begin{array}{cc}
1 & -1 \\
-1 & 1
\end{array}\right)
$$

Remark 1 If $g$ belongs to $\Gamma(1-i)$ then $\jmath(g)$ belongs to $S p_{6}(\mathbb{Z})$. Moreover, $\jmath(g)$ belongs to $S p_{6}(\mathbb{Z})$ for $g \in \Gamma$ if and only if $s(g)$ belongs to $G \subset S_{8}$.

We put

$$
\Gamma(G)=s^{-1}(G) \subset \Gamma, \quad S p_{6}(G)=\jmath(\Gamma(G)) \subset S p_{6}(\mathbb{Z}) .
$$

\subsection{5 automorphic forms $f_{J}$}

A holomorphic function $f$ on $\mathbb{B}^{5}$ is called an automorphic form of weight $k$ with respect to a finite index subgroup $\Gamma^{\prime}$ of $\Gamma$ if $f$ satisfies

$$
f(g \cdot y)=\psi(g, y)^{k} f(y)
$$

for any $g \in \Gamma^{\prime}$, where

$$
\psi(g, y)=\frac{{ }^{t}(T g y) U(T g y)}{{ }^{t}(T y) U(T y)} .
$$

Note that $\psi(g, y)=\operatorname{det}(g) \Psi(\jmath(g), \imath(y))$ by Lemma 1 .

We construct automorphic forms on $\mathbb{B}^{5}$ by using the pull-back $\vartheta_{a, b}(y)$ of theta constants $\vartheta_{a, b}(\tau)$ on $\mathbb{S}^{6}$ under the embedding $\imath: \mathbb{B}^{5} \rightarrow \mathbb{S}^{6}$.

Fact 3 The function $\prod_{j=0}^{3} \vartheta_{m_{j}, m_{j} U}(y)$ is an automorphic form on $\mathbb{B}^{5}$ of weight 2 with respect to $\Gamma(1-i)$, where

$$
\begin{array}{ll}
m_{0}=(0,0,0,0,0,0), & m_{1}=(0,0,1,1,1,1), \\
m_{2}=(1,1,0,0,1,1), & m_{3}=(1,1,1,1,0,0) .
\end{array}
$$


We define an action of $\Gamma$ on an automorphic form $f$ on $\mathbb{B}^{5}$ of weight $k$ with respect to $\Gamma(1-i)$ by

$$
f^{g}(y)=\psi(g, y)^{-k} f(g \cdot y) .
$$

\section{Fact 4}

(1) If $m U^{t} m \not \equiv 0 \bmod 4$ then $\vartheta_{m, m U}(y)=0$ for $m \in \mathbb{Z}^{6}$.

(2) (Corollary 4.11 in [MT]) The functions $\vartheta_{m, m U}(y)$ satisfy the quadratic relation

$$
\exp \left(\frac{\pi i}{2} v_{1}^{t} U_{0}\right) \vartheta_{v_{1}, v_{1} U}(y) \vartheta_{v_{2}, v_{2} U}(y)+\vartheta_{0, \ldots, 0}(y) \vartheta_{U_{0}, U_{0}}(y)=0,
$$

where $U_{0}=(1,1,0,0,1,1), v_{2}=v_{1}-U_{0},(0, \ldots, 0) \neq v_{1} \in \mathbb{Z}^{6}$ and $v_{1} U^{t} v_{1} \equiv 0 \bmod 4$. Especially, we have

$$
\vartheta_{m_{0}, m_{0}}(y) \vartheta_{m_{2}, m_{2}}(y)=\vartheta_{m_{1}, m_{1}}(y) \vartheta_{m_{3}, m_{3}}(y) .
$$

(3) By Fact 1, the set of theta characteristics $\left\{\left(m_{0}, m_{0}\right), \ldots,\left(m_{3}, m_{3}\right)\right\}$ may change by the action of $M \in S p_{6}(G)$. In spite of this situation, the function $f_{1}(y)=\prod_{j=0}^{3} \vartheta_{m_{j}, m_{j} U}(y)$ is invariant modulo sign under the action $g \in \Gamma(G)$ by $(2)$.

Since $\Gamma / \Gamma(1-i)$ is isomorphic to $S_{8}$, we have 105 automorphic forms $f_{J}(y)$ by acting $\Gamma$ on the function $f_{J_{1}}(y)$, where $J$ is a $(2,2,2,2)$-partition corresponding to an element $G \backslash S_{8}$ represented by $s(g) \in S_{8}$ for $g \in \Gamma$. The following is a main result in $[\mathrm{MT}]$.

Fact 5 The polynomials $x_{J}$ and automorphic forms $f_{J}(y)$ are $S_{8}$-equivariant. The vectors $\left(\ldots, x_{J}, \ldots\right)$ and $\left(\ldots, f_{J}(y), \ldots\right)$ are proportional and the functions $f_{J}(y)$ satisfy the same algebraic relations those $x_{J}$ satisfy.

Few $f_{J}(y)$ are explicitly given in terms of $\vartheta_{a, b}(y)$ in [MT], since $\jmath(g)$ does not belongs to $S p_{6}(\mathbb{Z})$ for a general $g \in \Gamma$. We give representations of $f_{J}(y)$ in terms of $\vartheta_{a, b}(y)$ for any $J \in \mathcal{J}$ in Section 5.

\section{3. $\kappa(M)$ for some $M \in S p_{6}(\mathbb{Z})$}

Lemma 2 Let $\kappa(M)$ be the eighth-root of unity in Fact 1 for $M \in S p_{6}(G)$ $\subset S p_{6}(\mathbb{Z})$. The map

$$
S p_{6}(G) \ni M \mapsto \kappa(M)^{4} \in\{ \pm 1\}
$$


is a homomorphism.

Proof. Note that $\phi_{a, b}(M)$ in Fact 1 becomes 0 for any $M \in S p_{6}(\mathbb{Z})$ when $(a, b)=(0, \ldots, 0)$, and that the function $\Psi(M, \tau)=\operatorname{det}(C \tau+D)$ satisfies

$$
\Psi(L M, \tau)=\Psi(L, M \cdot \tau) \Psi(M, \tau) .
$$

Since we have

$$
\begin{aligned}
& \kappa(L M)^{4} \Psi(L M, \tau)^{2} \vartheta_{0, \ldots, 0}^{4}(\tau) \\
= & \vartheta_{(L M) \cdot(0, \ldots, 0)}^{4}((L M) \cdot \tau)=\vartheta_{L \cdot(M \cdot(0, \ldots, 0))}^{4}(L \cdot(M \cdot \tau)) \\
= & \kappa(L)^{4} \exp \left(2 \pi i \phi_{M \cdot(0, \ldots, 0)}(L)\right)^{4} \Psi(L, M \cdot \tau)^{2} \vartheta_{M \cdot(0, \ldots, 0)}^{4}(M \cdot \tau) \\
= & \kappa(L)^{4} \kappa(M)^{4} \exp \left(2 \pi i \phi_{M \cdot(0, \ldots, 0)}(L)\right)^{4} \\
& \quad \times \Psi(L, M \cdot \tau)^{2} \Psi(M, \tau)^{2} \vartheta_{0, \ldots, 0}^{4}(\tau),
\end{aligned}
$$

we show that $4 \phi_{M \cdot(0, \ldots, 0)}(L)$ belongs to $\mathbb{Z}$ for any $L, M \in S p_{6}(G)$. Let $(a, b)$ be $M \cdot(0, \ldots, 0)$ for $M=\left(\begin{array}{cc}A & B \\ -U B U & U A U\end{array}\right)$. Note that

$$
b=-\left(U B^{t} A U\right)_{0} \equiv\left(U A^{t} B U\right)_{0} \equiv\left(A^{t} B\right)_{0} U \equiv a U \bmod \mathbb{Z}^{6} .
$$

The four times of $\phi_{a, a U}(L)$ for $L=\left(\begin{array}{cc}C & D \\ -U D U & U C U\end{array}\right)$ is congruent to

$$
\frac{1}{2}\left(a^{t}(U C U) D^{t} a+(a U)^{t}(-U D U) C^{t}(a U)\right)=0
$$

$\operatorname{modulo} \mathbb{Z}$.

Proposition 1 Let $g$ be an element of $\Gamma(1-i)$. Then the constant $\kappa(M)^{4}$ for $M=\jmath(g) \in S p_{6}(\mathbb{Z})$ is 1 .

Proof. It is shown in $[\mathrm{MY}]$ that the group $\Gamma(1-i)$ is generated by unitary reflections of order 2 . We show that $\kappa(M)^{4}=1$ for any element $M \in S p_{6}(\mathbb{Z})$ of order 2 , which satisfies

$$
\left(\begin{array}{ll}
A & B \\
C & D
\end{array}\right)=M=M^{-1}=\left(\begin{array}{cc}
{ }^{t} D & -{ }^{t} B \\
-{ }^{t} C & { }^{t} A
\end{array}\right)
$$

Since we have

$$
\begin{aligned}
& \vartheta_{0, \ldots, 0}^{2}(\tau)=\vartheta_{M^{2} \cdot(0, \ldots, 0)}^{2}\left(M^{2} \cdot \tau\right)=\vartheta_{M \cdot(M \cdot(0, \ldots, 0))}^{2}(M \cdot(M \cdot \tau)) \\
= & \kappa(M)^{4} \exp \left(4 \pi i \phi_{M \cdot(0, \ldots, 0)}(M)\right) \vartheta_{0, \ldots, 0}^{2}(\tau),
\end{aligned}
$$


it is sufficient to show that $2 \phi_{M \cdot(0, \ldots, 0)}(M) \in \mathbb{Z}$. We put $M \cdot(0, \ldots, 0)=$ $(a, b)$, which is $\left(\left(C^{t} D\right)_{0},\left(A^{t} B\right)_{0}\right)$. Note that $M^{2}=I_{12}$, and

$$
\begin{aligned}
M \cdot(a, b) & =(a, b) M^{-1}+(a, b) \equiv(0, \ldots, 0) \bmod 2 \mathbb{Z}^{12}, \\
(a, b) M^{-1} & =\left(a^{t} D-b^{t} C,-a^{t} B+b^{t} A\right) \equiv(a, b) \bmod 2 \mathbb{Z}^{12} .
\end{aligned}
$$

Since ${ }^{t} B C-{ }^{t} D A=B{ }^{t} C-A^{t} D=-I_{6}$ by ${ }^{t} B=-B,{ }^{t} C=-C$ and ${ }^{t} D=A$, we have

$$
\begin{aligned}
& 2 \phi_{a, b}(M) \\
= & -\frac{1}{4}\left(a^{t} D B^{t} a-2 a^{t} B C^{t} b+b^{t} C A^{t} b\right)+\frac{1}{2}\left(a^{t} D-b^{t} C\right)^{t}\left(A^{t} B\right)_{0} \\
= & \frac{1}{4}\left[\left(a^{t} D-b^{t} C\right)^{t}\left(-a^{t} B+b^{t} A\right)+a\left({ }^{t} B C-{ }^{t} D A\right)^{t} b+2\left(a^{t} D-b^{t} C\right)^{t} b\right] \\
\equiv & \frac{1}{4}\left(a^{t} b-a^{t} b\right)+\frac{1}{2} a^{t} b=\frac{1}{2} a^{t} b \quad \bmod \mathbb{Z} .
\end{aligned}
$$

By the action of $M \in S p_{6}(\mathbb{Z})$, the characteristic $(0, \ldots, 0)$ should be transformed into an even characteristic $(a, b)$, which satisfies $a^{t} b \in 2 \mathbb{Z}$.

Remark 2 If $M_{j k}^{2} \in S p_{6}(\mathbb{Z})$ keeps the theta characteristic $(0, \ldots, 0)$ invariant then the constant $\kappa\left(M_{j k}^{2}\right)^{2}$ is -1 . Otherwise, $\kappa\left(M_{j k}^{2}\right)^{2}$ may take \pm 1 . For examples, $M_{j, j+1}^{2}=M_{j}^{2}$ keeps the theta characteristic $(0, \ldots, 0)$ invariant, the constant $\kappa\left(M_{j}^{2}\right)^{2}$ is -1 . The elements $M_{15}^{2}, M_{16}^{2}, M_{47}^{2}$ and $M_{48}^{2}$ do not keep $(0, \ldots, 0)$ invariant. By computational evaluations of both sides of

$$
\vartheta_{M_{j k}^{2} \cdot(0, \ldots, 0)}^{2}\left(M_{j k}^{2} \cdot\left(i I_{6}\right)\right)=\kappa\left(M_{j k}^{2}\right)^{2} \Psi\left(M_{j k}^{2}, i I_{6}\right) \vartheta_{(0, \ldots, 0)}^{2}\left(i I_{6}\right),
$$

we have $\kappa\left(M_{15}^{2}\right)^{2}=\kappa\left(M_{48}^{2}\right)^{2}=1$ and $\kappa\left(M_{16}^{2}\right)^{2}=\kappa\left(M_{47}^{2}\right)^{2}=-1$.

Remark 3 We have $\kappa(M)^{4}=(-1)^{r}$ for any element $M=\left(\begin{array}{ll}A & B \\ C & D\end{array}\right) \in$ $S p_{r}(\mathbb{Z})$ satisfying $M^{2}=-I_{2 r}$, since

$$
\operatorname{det}(C(M \cdot \tau)+D) \operatorname{det}(C \tau+D)=\operatorname{det}\left(-I_{r}\right)=(-1)^{r}
$$

for $\tau \in \mathbb{S}^{r}$.

Lemma 3 The constant $\kappa(M)^{2}$ is $i$ for $M=M_{1}, M_{3}, M_{5}$ and $M_{7}$. 
Proof. We have $M_{k} \cdot(0, \ldots, 0)=(0, \ldots, 0), M_{k} \cdot \tau_{k}^{\prime}=\tau_{k}^{\prime}$ and $\operatorname{det}\left(C_{k} \tau_{k}^{\prime}+\right.$ $\left.D_{k}\right)=-i$ for $k=1,3,5,7$, where $\tau_{1}^{\prime}=\tau_{7}^{\prime}=i I_{6}$ and

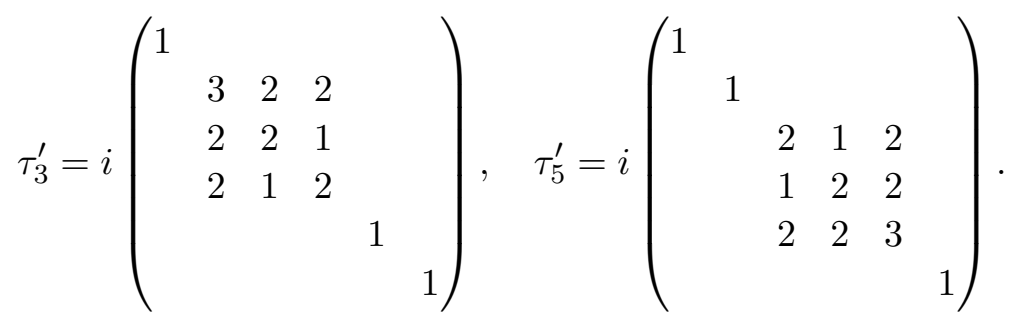

By Fact 1, we have

$$
\vartheta_{0, \ldots, 0}\left(\tau_{k}^{\prime}\right)=\kappa\left(M_{k}\right) \sqrt{-i} \vartheta_{0, \ldots, 0}\left(\tau_{k}^{\prime}\right),
$$

for $k=1,3,5,7$. Since $\vartheta_{0, \ldots, 0}\left(i \tau_{k}^{\prime}\right)$ are positive real numbers, $\kappa(g) \sqrt{-i}$ should be 1 for $g=M_{1}, M_{3}, M_{5}$ and $M_{7}$.

Lemma 4 The constant $\kappa(M)^{2}$ is -1 for $M=M_{14} M_{23}, M_{36} M_{45}$ and $M_{58} M_{67}$, where $M_{j k}=\jmath\left(g_{j k}\right)$.

Proof. For $M=M_{14} M_{23}=\left(\begin{array}{cc}A & B \\ C & D\end{array}\right)$, we put $g=A+i B U$. Its eigenvalues are 1 and $i$; the eigenspaces of 1 and $i$ are four and two dimensional, respectively. The element $T y={ }^{t}(1,1,1+i, 1+i, 0,0)$ satisfies $g(T y)=T y$ and $y \in \mathbb{B}^{5}$. By Lemma $1, \operatorname{det}(C \imath(y)+D)=1 / \operatorname{det}\left(T^{-1} g T\right)=-1$. By the property of the embedding $\imath: \mathbb{B}^{5} \rightarrow \mathbb{S}^{6}$, we have $(A \imath(y)+B)(C \imath(y)+D)^{-1}=$ $\imath\left(T^{-1} g T y\right)=\imath(y)$. Fact 1 implies that

$$
\vartheta_{(0, \ldots, 0)}^{2}(\imath(y))=\vartheta_{M \cdot(0, \ldots, 0)}^{2}(M \cdot \imath(y))=\kappa(M)^{2}(-1) \vartheta_{M \cdot(0, \ldots, 0)}^{2}(\imath(y)) .
$$

We show that $\vartheta_{0, \ldots, 0}(\imath(y))$ does not vanish by computational evaluations. Since

$$
\imath(y)=\left(\begin{array}{cc}
\tau^{\prime} & O \\
O & i I_{2}
\end{array}\right), \quad \tau^{\prime} \in \mathbb{S}^{4},
$$

we have

$$
\vartheta_{0, \ldots, 0}(\imath(y))=\vartheta_{0, \ldots, 0}\left(\tau^{\prime}\right) \vartheta_{0,0}^{2}(i) .
$$

The partial sum of the defining series of $\vartheta_{0, \ldots, 0}\left(\tau^{\prime}\right)$ for $n \in \mathbb{Z}^{4}$ satisfying $\operatorname{Im}\left(n \imath(y)^{t} n\right)<10$ is about $1.433725975+0.3384567922 i$, and $e^{-10 \pi}$ is about $0.2271101059 \times 10^{-13} \cdot 1.691709704+0.3997855731 i$ and $e^{-4 \pi}$ is about 
$0.3487342337 \times 10^{-5}$. These evaluations imply $\vartheta_{0, \ldots, 0}(\imath(y)) \neq 0$. Thus we have $\kappa(M)^{2}=-1$.

For $M_{36} M_{45}$ and $M_{58} M_{67}$, take $T y$ as

$$
{ }^{t}(0, i, 1+i, 1+i, 1,0), \quad{ }^{t}(0,0,1-i, 1-i, 1,1),
$$

respectively.

Lemmas 2, 3 and 4 imply the following.

Proposition 2 For $g \in \Gamma(G)$ and $y \in \mathbb{B}^{5}$, we have

$$
\begin{aligned}
& \operatorname{det}(g)^{2}=\kappa(\jmath(g))^{4}=\operatorname{sign}(s(g)), \\
& \kappa(\jmath(g))^{4} \Psi(\jmath(g), \imath(y))^{2}=\psi(g, y)^{2} .
\end{aligned}
$$

4. Transformation formulas of the theta constants for some elements of $S p_{6}(\mathbb{Q})$

Proposition 3 By the actions of $M_{2}, M_{4}, M_{6} \in S p_{6}(\mathbb{Q})$, we have

$$
\begin{aligned}
& \left(\begin{array}{c}
\vartheta_{a, b}\left(M_{2 k} \cdot \tau\right) \\
\vartheta_{a+e_{[2 k]}, b+e_{[2 k]}}\left(M_{2 k} \cdot \tau\right)
\end{array}\right) \\
= & \sqrt{\operatorname{det}\left(C_{2 k} \tau+D_{2 k}\right)} \exp \left(\pi i \frac{a_{2 k}-a_{2 k-1}}{2} \frac{b_{2 k}-b_{2 k-1}}{2}\right) \frac{1+i}{2} \\
& \times\left(\begin{array}{cc}
\exp \left(-\pi i \frac{a_{2 k}+a_{2 k-1}}{2}\right) & -\exp \left(\pi i \frac{a_{2 k}+a_{2 k-1}}{2}\right)
\end{array}\right) \\
1 & \times\left(\begin{array}{c}
\vartheta_{a^{\prime}, b^{\prime}+e_{[2 k]}}(\tau) \\
\vartheta_{a^{\prime}+e_{[2 k]}, b^{\prime}}(\tau)
\end{array}\right),
\end{aligned}
$$

where $\left(a^{\prime}, b^{\prime}\right)=-(a, b) M_{2 k}$,

$$
\begin{gathered}
e_{[2]}=(1,1,0,0,0,0), \quad e_{[4]}=(0,0,1,1,0,0), \\
e_{[6]}=(0,0,0,0,1,1),
\end{gathered}
$$

and the branch of the square root is determined by the assignment of the value at $\tau=i I_{6} \in \mathbb{S}^{6}$ as

$$
\sqrt{\operatorname{det}\left(C_{2 k}\left(i I_{6}\right)+D_{2 k}\right)}=\sqrt{-i}=\frac{1-i}{\sqrt{2}} .
$$


In order to prove this proposition, we prepare the following translation formula of theta constants for $\Delta=(1 / 2)\left(\begin{array}{cc}1 & -1 \\ -1 & 1\end{array}\right)$.

Lemma 5 Theta constants on the Siegel upper half space $\mathbb{S}^{2}$ of degree 2 satisfy

$$
\left(\begin{array}{c}
\vartheta_{a, b}(\tau+\Delta) \\
\vartheta_{a, b+e}(\tau+\Delta)
\end{array}\right)=\nabla(a)\left(\begin{array}{c}
\vartheta_{a, a \Delta+b}(\tau) \\
\vartheta_{a, a \Delta+b+e}(\tau)
\end{array}\right)
$$

where $e=(1,1)$, and

$$
\begin{aligned}
& \nabla(a)=\frac{c_{1}(a)}{2}\left(\begin{array}{cc}
1+i & (1-i) c_{2}^{-1}(a) \\
(1-i) c_{2}(a) & 1+i
\end{array}\right), \\
& c_{1}(a)=\exp \left(-\frac{\pi i a \Delta^{t} a}{4}\right), \quad c_{2}(a)=\exp \left(\frac{\pi i a^{t} e}{2}\right) .
\end{aligned}
$$

Proof. Let $\Lambda$ be the lattice $\left\{n=\left(n_{1}, n_{2}\right) \in \mathbb{Z}^{2} \mid n_{1}+n_{2} \in 2 \mathbb{Z}\right\}$. Note that $\left[\mathbb{Z}^{2}: \Lambda\right]=2$ and $\mathbb{Z}^{2} / \Lambda=\left\{(0,0), e_{1}=(1,0)\right\}$. We put

$$
\vartheta_{a, b}^{\Lambda}(\tau)=\sum_{n \in \Lambda} \exp \left(\pi i\left(n+\frac{a}{2}\right) \tau^{t}\left(n+\frac{a}{2}\right)+2 \pi i\left(n+\frac{a}{2}\right) \frac{t_{b}}{2}\right) .
$$

Since $n\left({ }^{t} e / 2\right)=\left(n_{1}+n_{2}\right) / 2 \in \mathbb{Z}$ for any $n \in \Lambda$, we have

$$
\vartheta_{a, b+e}^{\Lambda}(\tau)=\exp \left(\frac{\pi i a^{t} e}{2}\right) \vartheta_{a, b}^{\Lambda}(\tau)
$$

By definition, we have

$$
\vartheta_{a, b}(\tau)=\vartheta_{a, b}^{\Lambda}(\tau)+\vartheta_{a+2 e_{1}, b}^{\Lambda}(\tau)
$$

and

$$
\begin{aligned}
\vartheta_{a, b+e}(\tau) & =\vartheta_{a, b+e}^{\Lambda}(\tau)+\vartheta_{a+2 e_{1}, b+e}^{\Lambda}(\tau) \\
& =\exp \left(\frac{\pi i a^{t} e}{2}\right) \vartheta_{a, b}^{\Lambda}(\tau)+\exp \left(\frac{\pi i\left(a+2 e_{1}\right)^{t} e}{2}\right) \vartheta_{a+2 e_{1}, b}^{\Lambda}(\tau) \\
& =c_{2}(a)\left(\vartheta_{a, b}^{\Lambda}(\tau)-\vartheta_{a+2 e_{1}, b}^{\Lambda}(\tau)\right) .
\end{aligned}
$$

Thus we have

$$
\begin{aligned}
\left(\begin{array}{c}
\vartheta_{a, b}(\tau) \\
\vartheta_{a, b+e}(\tau)
\end{array}\right) & =\left(\begin{array}{cc}
1 & 1 \\
c_{2}(a) & -c_{2}(a)
\end{array}\right)\left(\begin{array}{c}
\vartheta_{a, b}^{\Lambda}(\tau) \\
\vartheta_{a+2 e_{1}, b}^{\Lambda}(\tau)
\end{array}\right), \\
\left(\begin{array}{c}
\vartheta_{a, b}^{\Lambda}(\tau) \\
\vartheta_{a+2 e_{1}, b}^{\Lambda}(\tau)
\end{array}\right) & =\frac{1}{2}\left(\begin{array}{cc}
1 & c_{2}^{-1}(a) \\
1 & -c_{2}^{-1}(a)
\end{array}\right)\left(\begin{array}{c}
\vartheta_{a, b}(\tau) \\
\vartheta_{a, b+e}(\tau)
\end{array}\right) .
\end{aligned}
$$


We express $\vartheta_{a, b}^{\Lambda}(\tau+\Delta)$ in terms of $\vartheta_{a^{\prime}, b^{\prime}}^{\Lambda}(\tau)$. Since

$$
\begin{aligned}
& \exp \left(\pi i\left(n+\frac{a}{2}\right) \Delta^{t}\left(n+\frac{a}{2}\right)\right) \\
= & \exp \left(\pi i n \Delta^{t} n\right) \exp \left(-\frac{\pi i a \Delta^{t} a}{4}\right) \exp \left(2 \pi i\left(n+\frac{a}{2}\right) \Delta \frac{{ }^{t} a}{2}\right) \\
= & \begin{cases}c_{1}(a) \exp \left(2 \pi i\left(n+\frac{a}{2}\right) \Delta \frac{{ }^{t} a}{2}\right) & \text { if } n \in \Lambda, \\
i c_{1}(a) \exp \left(2 \pi i\left(n+\frac{a}{2}\right) \Delta \frac{{ }^{t} a}{2}\right) & \text { if } n \in \Lambda+e_{1},\end{cases} \\
= & \sum_{n \in \Lambda}^{\Lambda}(\tau+\Delta) \\
\quad & \times \exp \left(\pi i\left(n+\frac{a}{2}\right) \tau^{t}\left(n+\frac{a}{2}\right)+2 \pi i\left(n+\frac{a}{2}\right) \frac{{ }^{t} b}{2}\right) \\
= & c_{1}(a) \sum_{n \in \Lambda} \exp \left(\pi i\left(n+\frac{a}{2}\right) \Delta^{t}\left(n+\frac{a}{2}\right)\right) \\
& \times \exp \left(2 \pi i\left(n+\frac{a}{2}\right) \tau^{t}\left(n+\frac{a}{2}\right)+2 \pi i\left(n+\frac{a}{2}\right) \frac{{ }^{t} b}{2}\right)
\end{aligned}
$$

Similarly, $\vartheta_{a+2 e_{1}, b}^{\Lambda}(\tau+\Delta)=i c_{1}(a) \vartheta_{a+2 e_{1}, a \Delta+b}^{\Lambda}(\tau)$. Thus we have

$$
\left(\begin{array}{c}
\vartheta_{a, b}^{\Lambda}(\tau+\Delta) \\
\vartheta_{a+2 e_{1}, b}^{\Lambda}(\tau+\Delta)
\end{array}\right)=c_{1}(a)\left(\begin{array}{cc}
1 & 0 \\
0 & i
\end{array}\right)\left(\begin{array}{c}
\vartheta_{a, b+a \Delta}^{\Lambda}(\tau) \\
\vartheta_{a+2 e_{1}, b+a \Delta}^{\Lambda}(\tau)
\end{array}\right) .
$$

Let us compute $\vartheta_{a, b}(\tau+\Delta)$ and $\vartheta_{a, b+e}(\tau+\Delta)$ :

$$
\begin{aligned}
& \left(\begin{array}{c}
\vartheta_{a, b}(\tau+\Delta) \\
\vartheta_{a, b+e}(\tau+\Delta)
\end{array}\right)=\left(\begin{array}{cc}
1 & 1 \\
c_{2}(a) & -c_{2}(a)
\end{array}\right)\left(\begin{array}{c}
\vartheta_{a, b}^{\Lambda}(\tau+\Delta) \\
\vartheta_{a+2 e_{1}, b}^{\Lambda}(\tau+\Delta)
\end{array}\right) \\
& =c_{1}(a)\left(\begin{array}{cc}
1 & 1 \\
c_{2}(a) & -c_{2}(a)
\end{array}\right)\left(\begin{array}{ll}
1 & 0 \\
0 & i
\end{array}\right)\left(\begin{array}{c}
\vartheta_{a, b+a \Delta}^{\Lambda}(\tau) \\
\vartheta_{a+2 e_{1}, b+a \Delta}^{\Lambda}(\tau)
\end{array}\right) \\
& =\frac{c_{1}(a)}{2}\left(\begin{array}{cc}
1 & 1 \\
c_{2}(a) & -c_{2}(a)
\end{array}\right)\left(\begin{array}{ll}
1 & 0 \\
0 & i
\end{array}\right)\left(\begin{array}{cc}
1 & c_{2}^{-1}(a) \\
1 & -c_{2}^{-1}(a)
\end{array}\right)\left(\begin{array}{c}
\vartheta_{a, b+a \Delta}(\tau) \\
\vartheta_{a, b+a \Delta+e}(\tau)
\end{array}\right),
\end{aligned}
$$

which implies this lemma. 
Proof of Proposition 3. It is sufficient to prove that

$$
\begin{aligned}
\left(\begin{array}{c}
\vartheta_{a, b}(M \cdot \tau) \\
\vartheta_{a+e, b+e}(M \cdot \tau)
\end{array}\right)= & \sqrt{\operatorname{det}\left(I_{2}-\Delta \tau-\Delta\right)} \exp \left(\pi i \frac{a_{2}-a_{1}}{2} \frac{b_{2}-b_{1}}{2}\right) \\
& \times \frac{1+i}{2}\left(\begin{array}{cc}
c_{2}^{-1}(a) & 1 \\
1 & -c_{2}(a)
\end{array}\right)\left(\begin{array}{c}
\vartheta_{a^{\prime}, b^{\prime}+e}(\tau) \\
\vartheta_{a^{\prime}+e, b^{\prime}}(\tau)
\end{array}\right),
\end{aligned}
$$

where $\tau \in \mathbb{S}^{2}, e=(1,1),\left(a^{\prime}, b^{\prime}\right)=(a, b) M, c_{2}(a)=\exp \left(\pi i\left(a_{1}+a_{2}\right) / 2\right)$,

$$
M=\left(\begin{array}{cc}
I_{2}-\Delta & \Delta \\
-\Delta & I_{2}-\Delta
\end{array}\right) \in S p_{2}(\mathbb{Q}), \quad \Delta=\frac{1}{2}\left(\begin{array}{cc}
1 & -1 \\
-1 & 1
\end{array}\right),
$$

and the branch of $\sqrt{\operatorname{det}\left(I_{2}-\Delta \tau-\Delta\right)}$ is determined by the assignment of the value at $\tau=i I_{2}$ as $\sqrt{-i}=(1-i) / \sqrt{2}$.

Since we have

$$
M=-P Q P Q P, \quad P=-\left(\begin{array}{cc}
I_{2} & \Delta \\
O_{2} & I_{2}
\end{array}\right), \quad Q=\left(\begin{array}{cc}
O_{2} & -I_{2} \\
I_{2} & O_{2}
\end{array}\right),
$$

use Lemma 5 and Fact 1 for $Q$ repeatedly. In this calculation, it is convenient to prepare formulas

$$
\begin{aligned}
& \left(\begin{array}{c}
\vartheta_{a, b}(\tau+\Delta) \\
\vartheta_{a, b+e}(\tau+\Delta) \\
\vartheta_{a+e, b}(\tau+\Delta) \\
\vartheta_{a+e, b+e}(\tau+\Delta)
\end{array}\right)=\Phi_{P}[a, b]\left(\begin{array}{c}
\vartheta_{a, a \Delta+b}(\tau) \\
\vartheta_{a, a \Delta+b+e}(\tau) \\
\vartheta_{a+e, a \Delta+b}(\tau) \\
\vartheta_{a+e, a \Delta+b+e}(\tau)
\end{array}\right), \\
& \left(\begin{array}{c}
\vartheta_{a, b}\left(-\tau^{-1}\right) \\
\vartheta_{a, b+e}\left(-\tau^{-1}\right) \\
\vartheta_{a+e, b}\left(-\tau^{-1}\right) \\
\vartheta_{a+e, b+e}\left(-\tau^{-1}\right)
\end{array}\right)=\Phi_{Q}[a, b]\left(\begin{array}{c}
\vartheta_{b,-a}(\tau) \\
\vartheta_{b,-a+e}(\tau) \\
\vartheta_{b+e,-a}(\tau) \\
\vartheta_{b+e,-a+e}(\tau)
\end{array}\right),
\end{aligned}
$$

where

$$
\begin{aligned}
& \Phi_{P}[a, b]=\left(\begin{array}{ll}
\nabla(a) & \\
& \nabla(a+e)
\end{array}\right) \\
& \Phi_{Q}[a, b]=\kappa(Q) \sqrt{\operatorname{det}(\tau)} \exp \left(\frac{\pi i a^{t} b}{2}\right)
\end{aligned}
$$




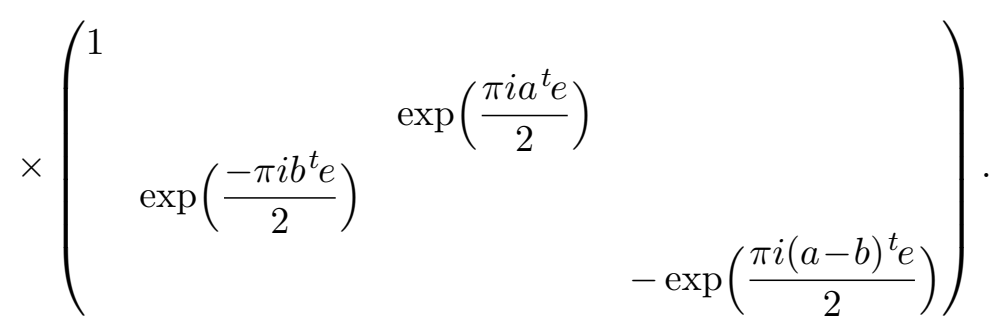

Since $(a, b) P=(a, a \Delta+b)$, we have

$\Phi_{P}[a, b] \Phi_{Q}[(a, b) P] \Phi_{P}[(a, b) P Q] \Phi_{Q}[(a, b) P Q P] \Phi_{P}[(a, b) P Q P Q]$

$$
=c(a, b) \frac{1+i}{2}\left(\begin{array}{cccc}
0 & c_{2}(a) & 1 & 0 \\
c_{2}(a) & 0 & 0 & c_{2}^{2}(a) \\
1 & 0 & 0 & -c_{2}(a) \\
0 & c_{2}^{2}(a) & -c_{2}(a) & 0
\end{array}\right)=\Phi_{-M}[a, b],
$$

where $c_{2}(a)=\exp \left(\pi i a^{t} e / 2\right)$ and

$$
c(a, b)=\kappa(Q)^{2} \sqrt{\operatorname{det}\left(I_{2}-\Delta \tau-\Delta\right)} \exp \left(\pi i \frac{a_{2}-a_{1}}{2} \frac{b_{2}-b_{1}}{2}\right) .
$$

Note that the theta characteristic $(a, b)$ is transformed into $-\left(a^{\prime}, b^{\prime}\right)=$ $-M(a, b)$ by $P Q P Q P$. We have

$$
\begin{aligned}
& \left(\begin{array}{c}
\vartheta_{a, b}(M \cdot \tau) \\
\vartheta_{a, b+e}(M \cdot \tau) \\
\vartheta_{a+e, b}(M \cdot \tau) \\
\vartheta_{a+e, b+e}(M \cdot \tau)
\end{array}\right)=\left(\begin{array}{c}
\vartheta_{a, b}(-M \cdot \tau) \\
\vartheta_{a, b+e}(-M \cdot \tau) \\
\vartheta_{a+e, b}(-M \cdot \tau) \\
\vartheta_{a+e, b+e}(-M \cdot \tau)
\end{array}\right) \\
& =\Phi_{-M}[a, b]\left(\begin{array}{c}
\vartheta_{-a^{\prime},-b^{\prime}}(\tau) \\
\vartheta_{-a^{\prime},-b^{\prime}+e}(\tau) \\
\vartheta_{-a^{\prime}+e,-b^{\prime}}(\tau) \\
\vartheta_{-a^{\prime}+e,-b^{\prime}+e}(\tau)
\end{array}\right) \\
& =c(a, b)\left(\begin{array}{cccc}
0 & c_{2}^{-1}(a) & 1 & 0 \\
c_{2}(a) & 0 & 0 & 1 \\
1 & 0 & 0 & -c_{2}^{-1}(a) \\
0 & 1 & -c_{2}(a) & 0
\end{array}\right)\left(\begin{array}{c}
\vartheta_{a^{\prime}, b^{\prime}}(\tau) \\
\vartheta_{a^{\prime}, b^{\prime}+e}(\tau) \\
\vartheta_{a^{\prime}+e, b^{\prime}}(\tau) \\
\vartheta_{a^{\prime}+e, b^{\prime}+e}(\tau)
\end{array}\right) \text {, }
\end{aligned}
$$

since $a^{\prime t} e=(a-(a+b) \Delta)^{t} e=a^{t} e$, and $\vartheta_{a^{\prime}, b^{\prime}-e}(\tau)=\exp \left(-\pi i a^{\prime t} e\right) \vartheta_{a^{\prime}, b^{\prime}+e}(\tau)$, $\vartheta_{a^{\prime}-e, b^{\prime}}(\tau)=\vartheta_{a^{\prime}+e, b^{\prime}}(\tau), \vartheta_{a^{\prime}-e, b^{\prime}-e}(\tau)=\exp \left(-\pi i a^{\prime} e\right) \vartheta_{a^{\prime}+e, b^{\prime}+e}(\tau)$. Especially, 


$$
\begin{aligned}
\left(\begin{array}{c}
\vartheta_{a, b}(M \cdot \tau) \\
\vartheta_{a+e, b+e}(M \cdot \tau)
\end{array}\right)= & \kappa(Q)^{2} \sqrt{\operatorname{det}\left(I_{2}-\Delta \tau-\Delta\right)} \\
& \times \exp \left(\frac{\pi i}{4}\left(a_{2}-a_{1}\right)\left(b_{2}-b_{1}\right)\right) \\
& \times \frac{1+i}{2}\left(\begin{array}{cc}
c_{2}^{-1}(a) & 1 \\
1 & -c_{2}(a)
\end{array}\right)\left(\begin{array}{l}
\vartheta_{a^{\prime}, b^{\prime}+e}(\tau) \\
\vartheta_{a^{\prime}+e, b^{\prime}}(\tau)
\end{array}\right)
\end{aligned}
$$

We have only to determine $\kappa(Q)^{2} \sqrt{\operatorname{det}\left(I_{2}-\Delta \tau-\Delta\right)}$ explicitly. The last equality for $\tau=i I_{2}$ and $a=b=(0,0)$ becomes

$$
\left(\begin{array}{l}
\vartheta_{00,00}\left(i I_{2}\right) \\
\vartheta_{11,11}\left(i I_{2}\right)
\end{array}\right)=\kappa(Q)^{2} \sqrt{-i} \frac{1+i}{2}\left(\begin{array}{cc}
1 & 1 \\
1 & -1
\end{array}\right)\left(\begin{array}{l}
\vartheta_{00,11}\left(i I_{2}\right) \\
\vartheta_{11,00}\left(i I_{2}\right)
\end{array}\right),
$$

since we have $M \cdot\left(i I_{2}\right)=i I_{2}$ and $\operatorname{det}\left(I_{2}-\Delta \tau-\Delta\right)=-i$. Note that $\vartheta_{11,11}\left(i I_{2}\right)=\vartheta_{1,1}(i) \vartheta_{1,1}(i)=0$, which implies $\vartheta_{00,11}\left(i I_{2}\right)=\vartheta_{11,00}\left(i I_{2}\right)$ and

$$
\vartheta_{00,00}\left(i I_{2}\right)=\kappa(Q)^{2} \sqrt{-i}(1+i) \vartheta_{11,00}\left(i I_{2}\right) .
$$

Since both of $\vartheta_{00,00}\left(i I_{2}\right)$ and $\vartheta_{11,00}\left(i I_{2}\right)$ are positive real numbers, $\kappa(Q)^{2} \sqrt{-i}$ should be $(1-i) / \sqrt{2}$. Thus if we choose the branch of the square root $\sqrt{\operatorname{det}\left(I_{2}-\Delta \tau-\Delta\right)}$ by the assignment of the value at $\tau=i I_{2}$ as $\sqrt{-i}=$ $(1-i) / \sqrt{2}$, then $\kappa(Q)^{2}=1$.

\section{Representations of $f_{J}(y)$}

In this section, we express $f_{J}(y)$ in terms of theta constants for all $(2,2,2,2)$-partitions $J$ by acting $S_{8}$ on $f_{J_{1}}(y)$. In subsection 5.1 , we explain an efficient method to get the representations for all forms $f_{J}(y)$, and in Subsections $5.2, \ldots, 5.5$, we list them.

\subsection{An efficient method to get the representations}

The following elementary lemma is a key to give 105 representations of $f_{J}(y)$.

Lemma 6 The double quotient $G \backslash S_{8} / G$ consists of five element, which are represented by

$$
\begin{gathered}
\sigma_{1}=\mathrm{id}, \sigma_{2}=(23), \sigma_{3}=(23)(45), \sigma_{4}=(23)(67), \\
\sigma_{5}=(23)(45)(67) .
\end{gathered}
$$

The cardinalities of the right $G$-orbits of $\sigma_{1}, \ldots, \sigma_{5}$ are $1,12,32,12$ and 48, respectively. 
We define an action of $\sigma \in S_{8}$ on an automorphic form $f(y)$ on $\mathbb{B}^{5}$ with respect to $\Gamma(1-i)$ by $f^{\sigma}(y)=f^{g}(y)$, where $g \in \Gamma$ satisfies $s(g)=\sigma$. We give $f_{J}$ by the action $\sigma \in S_{8}$ on $f_{J_{1}}=\prod_{j=0}^{4} \vartheta_{m_{j}}(y)$ corresponding to the polynomial $x_{J_{1}}$, where $m_{j}$ are in the following table.

\begin{tabular}{|c|c|cc|}
\hline$\#$ & $x_{J_{1}}$ & $m_{0}$ & $m_{1}$ \\
& & $m_{2}$ & $m_{3}$ \\
\hline \multirow{2}{*}{1} & $x_{\langle 12 ; 34 ; 56 ; 78\rangle}$ & 000000,000000 & 001111,001111 \\
& & 110011,110011 & 111100,111100 \\
\hline
\end{tabular}

We put $f_{J_{j}}(y)=f_{J_{1}}^{\sigma_{j}}(y)$ for $j=2, \ldots, 5$, which are

$$
\begin{aligned}
f_{J_{2}}(y) & =\prod_{j=0}^{3} \vartheta_{m_{j}, m_{j} U}^{g_{2}}(y)=\prod_{j=0}^{3} \vartheta_{m_{j}, m_{j} U}\left(M_{2} \cdot \imath(y)\right) / \psi\left(g_{2}, y\right)^{2}, \\
f_{J_{3}}(y) & =\prod_{j=0}^{3} \vartheta_{m_{j}, m_{j} U}^{g_{2} g_{4}}(y)=\prod_{j=0}^{3} \vartheta_{m_{j}, m_{j} U}\left(\left(M_{2} M_{4}\right) \cdot \imath(y)\right) / \psi\left(g_{2} g_{4}, y\right)^{2} \\
f_{J_{4}}(y) & =\prod_{j=0}^{3} \vartheta_{m_{j}, m_{j} U}^{g_{2} g_{6}}(y)=\prod_{j=0}^{3} \vartheta_{m_{j}, m_{j} U}\left(\left(M_{2} M_{6}\right) \cdot \imath(y)\right) / \psi\left(g_{2} g_{6}, y\right)^{2}, \\
f_{J_{5}}(y) & =\prod_{j=0}^{3} \vartheta_{m_{j}, m_{j} U}^{g_{2} g_{4} g_{6}}(y) \\
& =\prod_{j=0}^{3} \vartheta_{m_{j}, m_{j} U}\left(\left(M_{2} M_{4} M_{6}\right) \cdot \imath(y)\right) / \psi\left(g_{2} g_{4} g_{6}, y\right)^{2} .
\end{aligned}
$$

We express $f_{J_{j}}(y)$ in terms of theta constants by Proposition 4. We can express the rest one hundred $f_{J}(y)$ in terms of theta constants by the action of $G$ represented in $S p_{6}(\mathbb{Z})$ on $f_{J_{2}}(y), \ldots, f_{J_{5}}(y)$ by Lemma 6 . In this process, we have only to apply Fact 1.

The function $f_{J_{j}}^{\sigma}(y)$ for $\sigma \in G$ is independent of the choice of $g \in$ $\Gamma(G)$ such that $s(g)=\sigma$, but its representation in terms of theta constants $\vartheta_{a, b}(y)$ depends on the choice of $g$. Different representations of $f_{J_{j}}^{\sigma}(y)$ imply relations among $\vartheta_{a, b}(y)$.

At the end of this subsection, we give a lemma, which helps us to simplify the representations of $f_{J}(y)$. 
Lemma 7 For $y \in \mathbb{B}^{5}$ and $a, b \in \mathbb{Z}^{6}$, we have

$$
\vartheta_{(b U, a U)}(y)=\exp \left(-\pi i \frac{a^{t} b}{2}\right) \vartheta_{a, b}(y)
$$

Proof. Since $\imath(y)=\imath\left(i I_{6} y\right)$ and $\jmath\left(i I_{6}\right)=\left({ }_{-U} U\right)=V$, Fact 1 implies

$$
\begin{aligned}
& \vartheta_{(b U, a U)}(y)=\vartheta_{(b U,-a U)}(y)=\vartheta_{V \cdot(a, b)}(V \cdot \imath(y)) \\
= & \kappa(V) \exp \left(-\pi i \frac{a^{t} b}{2}\right) \sqrt{\operatorname{det}(-U \imath(y))} \vartheta_{a, b}(y) .
\end{aligned}
$$

Note that $\operatorname{det}(-U \imath(y))=-1$ and that $\vartheta_{0, \ldots, 0}(y)$ is not identically zero. Thus $\kappa(V) \sqrt{\operatorname{det}(-U \imath(y))}$ should be 1 .

\subsection{The $G$-orbit of $f_{J_{2}}(y)$}

\section{Proposition 4 We have}

$$
\begin{aligned}
f_{J_{2}}(y)=4 \vartheta_{110000,000000}(y) \vartheta_{111111,001111}(y) & \\
& \times \vartheta_{000011,110011}(y) \vartheta_{001100,111100}(y) .
\end{aligned}
$$

Proof. Proposition 4 implies

$$
\begin{aligned}
& \vartheta_{000000,000000}\left(M_{2} \cdot \tau\right) \\
& =\frac{1+i}{2} \sqrt{\Psi\left(M_{2}, \tau\right)}\left[\vartheta_{110000,000000}(\tau)+\vartheta_{000000,110000}(\tau)\right], \\
& \vartheta_{110000,110000}\left(M_{2} \cdot \tau\right) \\
& =\frac{1+i}{2} \sqrt{\Psi\left(M_{2}, \tau\right)}\left[\vartheta_{110000,000000}(\tau)-\vartheta_{000000,110000}(\tau)\right] .
\end{aligned}
$$

By Fact $4(1), \vartheta_{110000,110000}(y)$ is identically zero on $\mathbb{B}^{5}$, which implies

$$
\vartheta_{110000,000000}(y)=\vartheta_{000000,110000}(y) .
$$

Thus we have

$$
\vartheta_{000000,000000}\left(g_{2} \cdot y\right)=(1+i) \sqrt{\Psi\left(M_{2}, \imath(y)\right)} \vartheta_{110000,000000}(y) .
$$

Similarly, we have

$$
\begin{aligned}
& \vartheta_{001111,001111}\left(g_{2} \cdot y\right)=(1+i) \sqrt{\Psi\left(M_{2}, \imath(y)\right)} \vartheta_{111111,001111}(y), \\
& \vartheta_{110011,110011}\left(g_{2} \cdot y\right)=(1+i) \sqrt{\Psi\left(M_{2}, \imath(y)\right)} \vartheta_{000011,110011}(y), \\
& \vartheta_{111100,111100}\left(g_{2} \cdot y\right)=(1+i) \sqrt{\Psi\left(M_{2}, \imath(y)\right)} \vartheta_{001100,111100}(y) .
\end{aligned}
$$

Note that $\operatorname{det}\left(g_{2}\right)=i$ and that $\psi\left(g_{2}, y\right)=\operatorname{det}\left(g_{2}\right) \Psi\left(M_{2}, \imath(y)\right)$. 
By the action of $\sigma_{2}=(23) \in S_{8}$, the polynomial $x_{J_{1}}=x_{\langle 12 ; 34 ; 56 ; 78\rangle}$ changes into $x_{J_{2}}=x_{\langle 13 ; 24 ; 56 ; 78\rangle}$. The group

$$
G_{2}=\left\{\sigma \in G \mid x_{J_{2}}^{\sigma}= \pm x_{J_{2}}\right\}
$$

is generated by (56), (78), (12)(34), (13)(24) and (57)(68). Since its order is $2^{5}$, we have $\left[G: G_{2}\right]=12$. We express $f_{J_{2}}^{\sigma}(y)$ in terms of theta constants for $\sigma \in G_{2} \backslash G$.

Theorem 1 By the action of $\sigma \in G_{2} \backslash G, f_{J_{2}}(y)=\prod_{j=0}^{3} \vartheta_{\nu_{j}}(y)$ is transformed into

$$
f_{J_{2}}^{\sigma}(y)=4 \prod_{j=0}^{3} \vartheta_{\nu_{j}}^{g}(y)=4 \prod_{j=0}^{3}\left(\varepsilon_{j} \vartheta_{\mu_{j}}(y)\right)=\varepsilon 4 \prod_{j=0}^{3} \vartheta_{\mu_{j}}(y),
$$

where $\mu_{0}, \ldots, \mu_{3}, \varepsilon_{0}, \ldots, \varepsilon_{3}$, the sign $\varepsilon=\prod_{j=0}^{3} \varepsilon_{j}$ and $g \in \Gamma(G)$ such that $s(g)=\sigma$ are listed in the following table. In the table, $g_{j k}$ such that $s\left(g_{j k}\right)=$ $(j, k)$ are defined in the Section 2.3,

$$
\begin{array}{lll}
h_{12}=g_{13} g_{24}, & h_{13}=g_{15} g_{26}, & h_{14}=g_{17} g_{28}, \\
h_{23}=g_{35} g_{46}, & h_{24}=g_{37} g_{48}, & h_{34}=g_{57} g_{68},
\end{array}
$$

\begin{tabular}{|c|c|c|c|c|c|}
\hline \# & $g \in \Gamma(G)$ & $x_{J_{2}}^{\sigma}$ & $\varepsilon ; \begin{array}{l}\varepsilon_{0}, \varepsilon_{1} \\
\varepsilon_{0}, \varepsilon_{3}\end{array}$ & $\begin{array}{l}\mu_{0} \\
\mu_{2}\end{array}$ & $\begin{array}{l}\mu_{1} \\
\mu_{3}\end{array}$ \\
\hline \multirow[b]{2}{*}{2} & \multirow[b]{2}{*}{ id } & \multirow[b]{2}{*}{$x_{\langle 13 ; 24 ; 56 ; 78\rangle}$} & \multirow{2}{*}{$+; \begin{array}{l}1,1 \\
1,1\end{array}$} & $\frac{\mu_{2}}{110000,000000}$ & $\frac{\mu_{3}}{111111,001111}$ \\
\hline & & & & 000011,110011 & 001100,111100 \\
\hline \multirow{2}{*}{3} & \multirow[b]{2}{*}{$g_{1}$} & \multirow[b]{2}{*}{$x_{\langle 23 ; 14 ; 56 ; 78\rangle}$} & \multirow[b]{2}{*}{$+; \quad 1,1$} & 010000,100000 & 011111,101111 \\
\hline & & & & 100011,010011 & 101100,011100 \\
\hline \multirow{2}{*}{4} & \multirow{2}{*}{$h_{23}$} & \multirow{2}{*}{$x_{\langle 15 ; 26 ; 34 ; 78\rangle}$} & $\zeta, \zeta$ & 110110,000110 & 110101,000101 \\
\hline & & & $\zeta^{\prime}, \zeta^{\prime}$ & 000101,110101 & 000110,110110 \\
\hline \multirow{2}{*}{5} & \multirow{2}{*}{$g_{1} h_{23}$} & \multirow{2}{*}{$x_{\langle 25 ; 16 ; 34 ; 78\rangle}$} & $\zeta, \zeta$ & 010110,100110 & 010101,100101 \\
\hline & & & $\zeta^{\prime}, \zeta^{\prime}$ & 100101, 010101 & 100110,010110 \\
\hline \multirow{2}{*}{6} & \multirow{2}{*}{$h_{24}$} & \multirow[b]{2}{*}{$x_{\langle 17 ; 28 ; 56 ; 34\rangle}$} & $+i^{-i,-1}$ & 100001,011110 & 101110,010001 \\
\hline & & & $+;-i, 1$ & 010010, 101101 & 011101,100010 \\
\hline \multirow{2}{*}{7} & \multirow{2}{*}{$g_{1} h_{24}$} & \multirow{2}{*}{$x_{\langle 27 ; 18 ; 56 ; 34\rangle}$} & $+i,-1$ & 000001,111110 & 001110,110001 \\
\hline & & & $-i, 1$ & 110010,001101 & 111101,000010 \\
\hline
\end{tabular}

and $\zeta$ and $\zeta^{\prime}$ stand for $(1+i) / \sqrt{2}$ and $(1-i) / \sqrt{2}$, respectively. 


\begin{tabular}{|c|c|c|c|c|c|}
\hline 8 & $h_{13}$ & $x_{\langle 35 ; 46 ; 12 ; 78\rangle}$ & $+; \begin{array}{c}1, i \\
1,-i\end{array}$ & $\begin{array}{l}010010,011110 \\
100001,101101\end{array}$ & $\begin{array}{l}011101,010001 \\
101110,100010\end{array}$ \\
\hline 9 & $g_{1} h_{13}$ & $x_{\langle 36 ; 45 ; 12 ; 78\rangle}$ & $\begin{array}{r}1, i \\
1, i\end{array}$ & $\begin{array}{l}011000,011000 \\
101011,101011\end{array}$ & $\begin{array}{l}010111,010111 \\
100100,100100\end{array}$ \\
\hline 10 & $h_{14}$ & $x_{\langle 37 ; 48 ; 56 ; 12\rangle}$ & $+; \begin{array}{l}\zeta, \zeta^{\prime} \\
\zeta^{\prime}, \zeta\end{array}$ & $\begin{array}{l}001110,000001 \\
111101,110010\end{array}$ & $\begin{array}{l}111110,110001 \\
001101,000010\end{array}$ \\
\hline 11 & $g_{1} h_{14}$ & $x_{\langle 38 ; 47 ; 56 ; 12\rangle}$ & $+; \begin{array}{l}\zeta, \zeta^{\prime} \\
\zeta^{\prime}, \zeta\end{array}$ & $\begin{array}{l}001111,000000 \\
111100,110011\end{array}$ & $\begin{array}{l}111111,110000 \\
001100,000011\end{array}$ \\
\hline 12 & $h_{13} h_{24}$ & $x_{\langle 57 ; 68 ; 12 ; 34\rangle}$ & $+\begin{array}{l}1,1 \\
1,1\end{array}$ & $\begin{array}{l}000011,000000 \\
110000,110011\end{array}$ & $\begin{array}{l}001100,001111 \\
111111,111100\end{array}$ \\
\hline 13 & $g_{1} h_{13} h_{24}$ & $x_{\langle 67 ; 58 ; 12 ; 34\rangle}$ & $-; \begin{array}{c}1,1 \\
1,-1\end{array}$ & $\begin{array}{l}001001,000110 \\
111010,110101\end{array}$ & $\begin{array}{l}000110,001001 \\
110101,111010\end{array}$ \\
\hline
\end{tabular}

\subsection{The $G$-orbit of $f_{J_{3}}(y)$}

\section{Proposition 5 We have}

$$
f_{J_{3}}=\left(\vartheta_{111100,000000}^{2}-\vartheta_{110000,001100}^{2}\right)\left(\vartheta_{000011,111111}^{2}-\vartheta_{001111,110011}^{2}\right) .
$$

Proof. We get $f_{J_{3}}$ by $f_{J_{2}}^{g_{4}}$. Proposition 4 implies

$$
\begin{aligned}
& \vartheta_{110000,000000}\left(M_{4} \cdot \tau\right)=c\left(\vartheta_{110000,001100}(\tau)+\vartheta_{111100,000000}(\tau)\right), \\
& \vartheta_{111111,001111}\left(M_{4} \cdot \tau\right)=c\left(-\vartheta_{111111,000011}(\tau)+\vartheta_{110011,001111}(\tau)\right), \\
& \vartheta_{000011,110011}\left(M_{4} \cdot \tau\right)=c\left(\vartheta_{000011,111111}(\tau)+\vartheta_{001111,110011}(\tau)\right), \\
& \vartheta_{001100,111100}\left(M_{4} \cdot \tau\right)=c\left(-\vartheta_{001100,110000}(\tau)+\vartheta_{000000,111100}(\tau)\right),
\end{aligned}
$$

where $c=((1+i) / 2) \sqrt{\Psi\left(M_{4}, \tau\right)}$. Recall that $\operatorname{det}\left(g_{4}\right)=i$ and that $\psi\left(g_{4}, y\right)$ $=\operatorname{det}\left(g_{4}\right) \Psi\left(M_{4}, \imath(y)\right)$, and use Lemma 7 .

By the action of $\sigma_{3}=(23)(45) \in S_{8}$, the polynomial $x_{J_{1}}$ changes into $x_{J_{3}}=x_{\langle 13 ; 25 ; 46 ; 78\rangle}$. The group $G_{3}=\left\{\sigma \in G \mid x_{J_{3}}^{\sigma}= \pm x_{J_{3}}\right\}$ is generated by (78), (13)(24)(56) and (12)(35)(46). Since its order is 12, we have $\left[G: G_{3}\right]=32$. We express $f_{J_{3}}^{\sigma}(y)$ in terms of theta constants for $\sigma \in G_{3} \backslash G$.

Theorem 2 By the action of $\sigma \in G_{3} \backslash G$,

$$
f_{J_{3}}(y)=\left(\vartheta_{\nu_{0}}(y)^{2}-\vartheta_{\nu_{1}}(y)^{2}\right)\left(\vartheta_{\nu_{2}}(y)^{2}-\vartheta_{\nu_{3}}(y)^{2}\right)
$$

is transformed into

$$
\begin{aligned}
f_{J_{3}}^{\sigma}(y) & =\left(\vartheta_{\nu_{0}}^{g}(y)^{2}-\vartheta_{\nu_{1}}^{g}(y)^{2}\right)\left(\vartheta_{\nu_{2}}^{g}(y)^{2}-\vartheta_{\nu_{3}}^{g}(y)^{2}\right) \\
& =\left[\left(\varepsilon_{0} \vartheta_{\mu_{0}}(y)\right)^{2}-\left(\varepsilon_{1} \vartheta_{\mu_{1}}(y)\right)^{2}\right]\left[\left(\varepsilon_{2} \vartheta_{\mu_{2}}(y)\right)^{2}-\left(\varepsilon_{3} \vartheta_{\mu_{3}}^{g}(y)\right)^{2}\right]
\end{aligned}
$$




$$
=\varepsilon\left(\vartheta_{\mu_{0}}^{2}(y)+\varepsilon^{\prime} \vartheta_{\mu_{1}}^{2}(y)\right)\left(\vartheta_{\mu_{2}}^{2}(y)+\varepsilon^{\prime} \vartheta_{\mu_{3}}^{2}(y)\right),
$$

where $\mu_{0}, \ldots, \mu_{3}, \varepsilon_{0}, \ldots, \varepsilon_{3}$, the signs $\varepsilon, \varepsilon^{\prime}$ and $g \in \Gamma(G)$ such that $s(g)=$ $\sigma$ are listed in the following table.

\begin{tabular}{|c|c|c|c|c|c|}
\hline$\#$ & $g \in \Gamma(g)$ & $x_{J_{3}}^{\sigma}$ & $\begin{array}{l}\varepsilon \\
\varepsilon^{\prime} ; \begin{array}{l}\varepsilon_{0}, \\
\varepsilon_{2}, \\
\varepsilon_{2}\end{array}\end{array}$ & $\begin{array}{l}\mu_{0} \\
\mu_{2}\end{array}$ & $\begin{array}{l}\mu_{1} \\
\mu_{3}\end{array}$ \\
\hline 14 & id & $x_{\langle 13 ; 25 ; 46 ; 78\rangle}$ & $\begin{array}{ll}+ & 1,1 \\
- & 1,1\end{array}$ & 111100,000000 & 110000,001100 \\
\hline 15 & $g_{3}$ & $x_{\langle 14 ; 25 ; 36 ; 78\rangle}$ & $\begin{array}{ll}+ & 1, i \\
+ & 1, i\end{array}$ & 000011,111111 & 001111, 110011 \\
\hline 16 & $g_{1}$ & $x_{\langle 23 ; 15 ; 46 ; 78\rangle}$ & $\begin{array}{l}+ \\
+ \\
-\quad 1,1\end{array}$ & 011100, 100000 & 010000, 101100 \\
\hline 17 & $g_{1} g_{3}$ & $x_{\langle 24 ; 15 ; 36 ; 78\rangle}$ & $\begin{array}{ll}+ & 1, i \\
+ & 1, i\end{array}$ & 100011,011111 & 101111, 010011 \\
\hline 18 & $g_{5}$ & $x_{\langle 13 ; 26 ; 45 ; 78\rangle}$ & $\begin{array}{c}+ \\
+ \\
- \\
1,-1\end{array}$ & 110110,000110 & 111010,001010 \\
\hline 19 & $g_{3} g_{5}$ & $x_{\langle 14 ; 26 ; 35 ; 78\rangle}$ & $\begin{array}{c}+ \\
+ \\
+ \\
1,-i\end{array}$ & 001001, 111001 & 000101, 110101 \\
\hline 20 & $g_{1} g_{5}$ & $x_{\langle 23 ; 16 ; 45 ; 78\rangle}$ & $\begin{array}{c}+ \\
+ \\
- \\
1,-1\end{array}$ & 010110,100110 & 011010, 101010 \\
\hline 21 & $g_{1} g_{3} g_{5}$ & $x_{\langle 24 ; 16 ; 35 ; 78\rangle}$ & 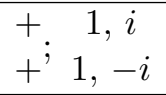 & 101001, 011001 & 100101, 010101 \\
\hline 22 & $h_{34}$ & $x_{\langle 13 ; 27 ; 48 ; 56\rangle}$ & $\begin{array}{l}+\zeta, \zeta \\
-\zeta^{\prime},-\zeta^{\prime}\end{array}$ & 111110, 000001 & 110001,001110 \\
\hline 23 & $g_{3} h_{34}$ & $x_{\langle 14 ; 27 ; 38 ; 56\rangle}$ & $\begin{array}{l}+\zeta,-\zeta^{\prime} \\
+\zeta^{\prime},-\zeta \\
\end{array}$ & 000010,111101 & 001101,110010 \\
\hline 24 & $g_{1} h_{34}$ & $x_{\langle 23 ; 17 ; 48 ; 56\rangle}$ & $\begin{array}{l}+\zeta, \zeta \\
{ }_{-} \zeta^{\prime},-\zeta^{\prime}\end{array}$ & 011110, 100001 & 010001, 101110 \\
\hline 25 & $g_{1} g_{3} h_{34}$ & $x_{\langle 24 ; 17 ; 38 ; 56\rangle}$ & $\begin{array}{l}+\zeta,-\zeta^{\prime} \\
+\zeta^{\prime},-\zeta\end{array}$ & 100010,011101 & 101101, 010010 \\
\hline 26 & $g_{5} h_{34}$ & $x_{\langle 13 ; 28 ; 47 ; 56\rangle}$ & $\begin{array}{l}+\zeta, \zeta \\
-\zeta^{\prime},-\zeta^{\prime}\end{array}$ & 111111,000000 & 110000,001111 \\
\hline 27 & $g_{3} g_{5} h_{34}$ & $x_{\langle 14 ; 28 ; 37 ; 56\rangle}$ & $\begin{array}{l}+\zeta,-\zeta^{\prime} \\
+\zeta^{\prime},-\zeta\end{array}$ & 000011,111100 & 001100,110011 \\
\hline 28 & $g_{1} g_{5} h_{34}$ & $x_{\langle 23 ; 18 ; 47 ; 56\rangle}$ & $\begin{array}{l}+\zeta, \zeta \\
-\zeta^{\prime},-\zeta^{\prime}\end{array}$ & 011111,100000 & 010000,101111 \\
\hline 29 & $g_{1} g_{3} g_{5} h_{34}$ & $x_{\langle 24 ; 18 ; 37 ; 56\rangle}$ & $\begin{array}{l}+\zeta,-\zeta^{\prime} \\
+\zeta^{\prime},-\zeta\end{array}$ & 100011,011100 & 101100,010011 \\
\hline
\end{tabular}




\begin{tabular}{|c|c|c|c|c|c|}
\hline 30 & $h_{24}$ & $-x_{\langle 17 ; 25 ; 68 ; 34\rangle}$ & $\begin{array}{l}-i,-1 \\
+;-1,-i\end{array}$ & 101010,011010 & 101001, 011001 \\
\hline 31 & $g_{3} h_{24}$ & $-x_{\langle 18 ; 25 ; 67 ; 34\rangle}$ & $\begin{array}{l}--i,-i \\
-;-1,1\end{array}$ & 011010,101010 & 011001,101001 \\
\hline 32 & $g_{1} h_{24}$ & $-x_{\langle 27 ; 15 ; 68 ; 34\rangle}$ & $\begin{array}{l}-,-i,-1 \\
+\quad-1,-i\end{array}$ & 001010,111010 & 001001,111001 \\
\hline 33 & $g_{1} g_{3} h_{24}$ & $-x_{\langle 28 ; 15 ; 67 ; 34\rangle}$ & $\begin{array}{l}-,-i,-i \\
-;-1,1\end{array}$ & 111010,001010 & 111001, 001001 \\
\hline 34 & $g_{5} h_{24}$ & $-x_{\langle 17 ; 26 ; 58 ; 34\rangle}$ & $\begin{array}{l}-,-i,-1 \\
+\quad-1,-i\end{array}$ & 100000,011100 & 100011,011111 \\
\hline 35 & $g_{3} g_{5} h_{24}$ & $-x_{\langle 18 ; 26 ; 57 ; 34\rangle}$ & $\begin{array}{l}-,-i,-i \\
-;-1,1\end{array}$ & 010000,101100 & 010011,101111 \\
\hline 36 & $g_{1} g_{5} h_{24}$ & $-x_{\langle 27 ; 16 ; 58 ; 34\rangle}$ & $\begin{array}{l}-,-i,-1 \\
+-1,-i\end{array}$ & 000000,111100 & 000011,111111 \\
\hline 37 & $g_{1} g_{3} g_{5} h_{24}$ & $-x_{\langle 28 ; 16 ; 57 ; 34\rangle}$ & $\begin{array}{l}--i,-i \\
-;-1,1\end{array}$ & 110000,001100 & 110011,001111 \\
\hline 38 & $h_{14}$ & $x_{\langle 37 ; 58 ; 46 ; 12\rangle}$ & $\begin{array}{l}+\zeta,-\zeta \\
-{ }^{\prime} \zeta^{\prime}, \zeta^{\prime}\end{array}$ & 000010,000001 & 001110,001101 \\
\hline 39 & $g_{3} h_{14}$ & $x_{\langle 47 ; 58 ; 36 ; 12\rangle}$ & $\begin{array}{c}+ \\
+ \\
+^{\prime} \\
-\zeta^{\prime}, \zeta\end{array}$ & 111101,111110 & 110001,110010 \\
\hline 40 & $g_{1} h_{14}$ & $x_{\langle 38 ; 57 ; 46 ; 12\rangle}$ & $\begin{array}{l}+\zeta,-\zeta \\
-^{\prime}-\zeta^{\prime}, \zeta^{\prime}\end{array}$ & 000011,000000 & 001111,001100 \\
\hline 41 & $g_{1} g_{3} h_{14}$ & $x_{\langle 48 ; 57 ; 36 ; 12\rangle}$ & $\begin{array}{c}+{ }^{+}, \zeta^{\prime} \\
+^{\prime}-\zeta^{\prime}, \zeta\end{array}$ & 111100,111111 & 110000,110011 \\
\hline 42 & $g_{5} h_{14}$ & $x_{\langle 37 ; 68 ; 45 ; 12\rangle}$ & $\begin{array}{c}+{ }^{+} \zeta, \zeta \\
-{ }^{\prime} \zeta^{\prime}, \zeta^{\prime}\end{array}$ & 001000,000111 & 000100,001011 \\
\hline 43 & $g_{3} g_{5} h_{14}$ & $x_{\langle 47 ; 68 ; 35 ; 12\rangle}$ & $\begin{array}{l}+\zeta,-\zeta^{\prime} \\
+{ }^{\prime}-\zeta^{\prime}, \zeta\end{array}$ & 110111,111000 & 111011,110100 \\
\hline 44 & $g_{1} g_{5} h_{14}$ & $x_{\langle 38 ; 67 ; 45 ; 12\rangle}$ & $\begin{array}{c}+ \\
+ \\
{ }^{+}-\zeta^{\prime}, \zeta^{\prime}\end{array}$ & 001001,000110 & 000101,001010 \\
\hline 45 & $g_{1} g_{3} g_{5} h_{14}$ & $x_{\langle 48 ; 67 ; 35 ; 12\rangle}$ & $\begin{array}{l}+\zeta,-\zeta^{\prime} \\
+{ }^{\prime}-\zeta^{\prime}, \zeta\end{array}$ & 110110,111001 & 111010,110101 \\
\hline
\end{tabular}

\subsection{The $G$-orbit of $f_{J_{4}}(y)$}

\section{Proposition 6 We have}

$$
f_{J_{4}}=\left(\vartheta_{110011,000000}^{2}-\vartheta_{110000,000011}^{2}\right)\left(\vartheta_{001100,111111}^{2}-\vartheta_{001111,111100}^{2}\right) .
$$

Proof. We get $f_{J_{4}}$ by $f_{J_{2}}^{g_{6}}$. Proposition 4 implies

$$
\vartheta_{110000,000000}\left(M_{6} \cdot \tau\right)=c\left(\vartheta_{110000,000011}(\tau)+\vartheta_{110011,000000}(\tau)\right),
$$




$$
\begin{aligned}
& \vartheta_{111111,001111}\left(M_{6} \cdot \tau\right)=c\left(-\vartheta_{111111,001100}(\tau)+\vartheta_{111100,001111}(\tau)\right), \\
& \vartheta_{000011,110011}\left(M_{6} \cdot \tau\right)=c\left(-\vartheta_{000011,110000}(\tau)+\vartheta_{000000,110011}(\tau)\right), \\
& \vartheta_{001100,111100}\left(M_{6} \cdot \tau\right)=c\left(\vartheta_{001100,111111}(\tau)+\vartheta_{001111,111100}(\tau)\right),
\end{aligned}
$$

where $c=((1+i) / 2) \sqrt{\Psi\left(M_{6}, \tau\right)}$. Use Lemma 7 and the equalities $\operatorname{det}\left(g_{6}\right)=$ $i$ and $\psi\left(g_{6}, y\right)=\operatorname{det}\left(g_{6}\right) \Psi\left(M_{6}, \imath(y)\right)$.

By the action of $\sigma_{4}=(67) \in S_{8}$, the polynomial $x_{J_{2}}=x_{\langle 13 ; 24 ; 56 ; 78\rangle}$ changes into $x_{J_{4}}=x_{\langle 13 ; 24 ; 57 ; 68\rangle}$. The group

$$
G_{4}=\left\{\sigma \in G \mid x \sigma_{J_{4}}= \pm x_{J_{4}}\right\}
$$

is generated by $(12)(34),(13)(24),(56)(78),(57)(68)$ and $(15)(26)(37)(48)$. Since its order is 32 , we have $\left[G: G_{4}\right]=12$. We express $f_{J_{4}}^{\sigma}(y)$ in terms of theta constants for $\sigma \in G_{4} \backslash G$.

Theorem 3 For $\sigma \in G_{4} \backslash G, f_{J_{4}}(y)=\left(\vartheta_{\nu_{0}}(y)^{2}-\vartheta_{\nu_{1}}(y)^{2}\right)\left(\vartheta_{\nu_{2}}(y)^{2}-\vartheta_{\nu_{3}}(y)^{2}\right)$ is transformed into

\begin{tabular}{|c|c|c|c|c|c|}
\hline$\#$ & $g \in \Gamma(G)$ & $x_{J_{4}}^{\sigma}$ & $\varepsilon^{\prime} ; \varepsilon_{0}, \varepsilon_{1}$ & $\mu_{0}$ & $\begin{array}{l}\mu_{1} \\
\mu_{3}\end{array}$ \\
\hline \multirow{2}{*}{46} & \multirow[b]{2}{*}{10} & \multirow[b]{2}{*}{$x_{\langle 13 ; 24 ; 57 ; 68\rangle}$} & 1,1 & 110011,000000 & 110000,000011 \\
\hline & & & $; 1,1$ & 001100,111111 & 001111, 111100 \\
\hline \multirow{2}{*}{47} & \multirow{2}{*}{$h_{23}$} & \multirow[b]{2}{*}{$x_{\langle 15 ; 26 ; 37 ; 48\rangle}$} & $-1, i$ & 111111,001100 & 110000,000011 \\
\hline & & & ${ }^{+} ; 1, i$ & 000000,110011 & 001111,111100 \\
\hline \multirow{2}{*}{48} & \multirow{2}{*}{$h_{24}$} & \multirow[b]{2}{*}{$x_{\langle 17 ; 28 ; 35 ; 46\rangle}$} & 1,1 & 110011,000000 & 111111,001100 \\
\hline & & & 1,1 & 000011,110000 & 001111,111100 \\
\hline \multirow{2}{*}{49} & & \multirow{2}{*}{$x_{\langle 23 ; 14 ; 57 ; 68\rangle}$} & 1,1 & 010011,100000 & 010000,100011 \\
\hline & & & 1,1 & 101100,011111 & 101111,011100 \\
\hline \multirow{2}{*}{50} & \multirow{2}{*}{$g_{1} h_{23}$} & \multirow[b]{2}{*}{$x_{\langle 25 ; 16 ; 37 ; 48\rangle}$} & $-1, i$ & 011111,101100 & 010000,100011 \\
\hline & & & $; 1, i$ & 100000,010011 & 101111, 011100 \\
\hline \multirow{2}{*}{51} & \multirow{2}{*}{$g_{1} h_{24}$} & \multirow[b]{2}{*}{$x_{\langle 27 ; 18 ; 35 ; 46\rangle}$} & 1,1 & 010011,100000 & 011111,101100 \\
\hline & & & 1,1 & 100011,010000 & 101111,011100 \\
\hline
\end{tabular}

$$
\begin{aligned}
f_{J_{4}}^{\sigma}(y) & =\left(\vartheta_{\nu_{0}}^{g}(y)^{2}-\vartheta_{\nu_{1}}^{g}(y)^{2}\right)\left(\vartheta_{\nu_{2}}^{g}(y)^{2}-\vartheta_{\nu_{3}}^{g}(y)^{2}\right) \\
& =\left[\left(\varepsilon_{0} \vartheta_{\mu_{0}}(y)\right)^{2}-\left(\varepsilon_{1} \vartheta_{\mu_{1}}(y)\right)^{2}\right]\left[\left(\varepsilon_{2} \vartheta_{\mu_{2}}(y)\right)^{2}-\left(\varepsilon_{3} \vartheta_{\mu_{3}}^{g}(y)\right)^{2}\right] \\
& =\left(\vartheta_{\mu_{0}}^{2}(y)+\varepsilon^{\prime} \vartheta_{\mu_{1}}^{2}(y)\right)\left(\vartheta_{\mu_{2}}^{2}(y)+\varepsilon^{\prime} \vartheta_{\mu_{3}}^{2}(y)\right)
\end{aligned}
$$

where $\mu_{0}, \ldots, \mu_{3}, \varepsilon_{0}, \ldots, \varepsilon_{3}$, the sign $\varepsilon^{\prime}$ and $g \in \Gamma(G)$ such that $s(g)=\sigma$ are listed in the following table. 


\begin{tabular}{|c|c|c|c|c|c|}
\hline 52 & $g_{7}$ & $x_{\langle 13 ; 24 ; 58 ; 67\rangle}$ & $-; \begin{array}{l}1,1 \\
1,1\end{array}$ & $\begin{array}{l}110010,000001 \\
001101,111110\end{array}$ & $\begin{array}{l}110001,000010 \\
001110,111101\end{array}$ \\
\hline 53 & $g_{7} h_{23}$ & $x_{\langle 15 ; 26 ; 38 ; 47\rangle}$ & $\begin{array}{c}-1, i \\
1, i\end{array}$ & $\begin{array}{l}111110,001101 \\
000001,110010\end{array}$ & $\begin{array}{l}110001,000010 \\
001110,111101\end{array}$ \\
\hline 54 & $g_{7} h_{24}$ & $x_{\langle 17 ; 28 ; 45 ; 36\rangle}$ & $-; \begin{array}{c}1,-1 \\
1,1\end{array}$ & $\begin{array}{l}101011,010100 \\
011011,100100\end{array}$ & $\begin{array}{l}100111,011000 \\
010111,101000\end{array}$ \\
\hline 55 & $g_{1} g_{7}$ & $x_{\langle 23 ; 14 ; 58 ; 67\rangle}$ & $-; \begin{array}{l}1,1 \\
1,1\end{array}$ & $\begin{array}{l}010010,100001 \\
101101,011110\end{array}$ & $\begin{array}{l}010001,100010 \\
101110,011101\end{array}$ \\
\hline 56 & $g_{1} g_{7} h_{23}$ & $x_{\langle 25 ; 16 ; 38 ; 47\rangle}$ & $\begin{array}{c}-1, i \\
1, i\end{array}$ & $\begin{array}{l}011110,101101 \\
100001,010010\end{array}$ & $\begin{array}{l}010001,100010 \\
101110,011101\end{array}$ \\
\hline 57 & $g_{1} g_{7} h_{24}$ & $x_{\langle 27 ; 18 ; 45 ; 36\rangle}$ & $-; \begin{array}{c}1,-1 \\
1,1\end{array}$ & $\begin{array}{l}001011,110100 \\
111011,000100\end{array}$ & $\begin{array}{l}000111,111000 \\
110111,001000\end{array}$ \\
\hline
\end{tabular}

\subsection{The $G$-orbit of $f_{J_{5}}(y)$}

\section{Proposition 7 We have}

$$
\begin{aligned}
f_{J_{5}}= & \frac{1}{4}\left(\vartheta_{111111,000000}+\vartheta_{110000,001111}+\vartheta_{001100,110011}+\vartheta_{000011,111100}\right) \\
& \times\left(\vartheta_{111111,000000}+\vartheta_{110000,001111}-\vartheta_{001100,110011}-\vartheta_{000011,111100}\right) \\
& \times\left(\vartheta_{111111,000000}-\vartheta_{110000,001111}+\vartheta_{001100,110011}-\vartheta_{000011,111100}\right) \\
& \times\left(\vartheta_{111111,000000}-\vartheta_{110000,001111}-\vartheta_{001100,110011}+\vartheta_{000011,111100}\right) .
\end{aligned}
$$

Proof. We get $f_{J_{5}}$ by $f_{J_{4}}^{g_{4}}$. Proposition 4 implies

$$
\begin{aligned}
& \vartheta_{110011,000000}\left(M_{4} \cdot \tau\right)=c\left(\vartheta_{110011,001100}(\tau)+\vartheta_{111111,000000}(\tau)\right), \\
& \vartheta_{110000,000011}\left(M_{4} \cdot \tau\right)=c\left(\vartheta_{110000,001111}(\tau)+\vartheta_{111100,000011}(\tau)\right), \\
& \vartheta_{001100,111111}\left(M_{4} \cdot \tau\right)=c\left(-\vartheta_{001100,110011}(\tau)+\vartheta_{000000,111111}(\tau)\right), \\
& \vartheta_{001111,111100}\left(M_{4} \cdot \tau\right)=c\left(-\vartheta_{001111,110000}(\tau)+\vartheta_{000011,111100}(\tau)\right),
\end{aligned}
$$

where $c=((1+i) / 2) \sqrt{\Psi\left(M_{4}, \tau\right)}$. Use Lemma 7 and the equalities $\operatorname{det}\left(g_{4}\right)=$ $i$ and $\psi\left(g_{4}, y\right)=\operatorname{det}\left(g_{4}\right) \Psi\left(M_{4}, \imath(y)\right)$.

By the action of $\sigma_{5}=(23)(45)(67) \in S_{8}$, the polynomial $x_{J_{1}}$ changes into $x_{J_{5}}=x_{\langle 13 ; 25 ; 47 ; 68\rangle}$. The group

$$
G_{5}=\left\{\sigma \in G \mid x_{J_{5}}^{\sigma}= \pm x_{J_{5}}\right\}
$$

is generated by $(13)(24)(57)(68),(16)(25)(38)(47)$ and $(17)(28)(34)(56)$. Since its order is 8 , we have $\left[G: G_{5}\right]=48$. We express $f_{J_{5}}^{\sigma}(y)$ in terms of theta constants for $\sigma \in G_{5} \backslash G$. 
Theorem 4 For $\sigma \in G_{5} \backslash G, f_{J_{5}}^{\sigma}(y)$ is

$$
\begin{aligned}
& \frac{1}{4}\left(\varepsilon_{0} \vartheta_{\mu_{0}}+\varepsilon_{1} \vartheta_{\mu_{1}}+\varepsilon_{2} \vartheta_{\mu_{2}}+\varepsilon_{3} \vartheta_{\mu_{3}}\right)\left(\varepsilon_{0} \vartheta_{\mu_{0}}+\varepsilon_{1} \vartheta_{\mu_{1}}-\varepsilon_{2} \vartheta_{\mu_{2}}-\varepsilon_{3} \vartheta_{\mu_{3}}\right) \\
& \times\left(\varepsilon_{0} \vartheta_{\mu_{0}}-\varepsilon_{1} \vartheta_{\mu_{1}}+\varepsilon_{2} \vartheta_{\mu_{2}}-\varepsilon_{3} \vartheta_{\mu_{3}}\right)\left(\varepsilon_{0} \vartheta_{\mu_{0}}-\varepsilon_{1} \vartheta_{\mu_{1}}-\varepsilon_{2} \vartheta_{\mu_{2}}+\varepsilon_{3} \vartheta_{\mu_{3}}\right) \\
& =\frac{\varepsilon}{4}\left(\vartheta_{\mu_{0}}+\varepsilon_{1}^{\prime} \vartheta_{\mu_{1}}+\varepsilon_{2}^{\prime} \vartheta_{\mu_{2}}+\varepsilon_{3}^{\prime} \vartheta_{\mu_{3}}\right)\left(\vartheta_{\mu_{0}}+\varepsilon_{1}^{\prime} \vartheta_{\mu_{1}}-\varepsilon_{2}^{\prime} \vartheta_{\mu_{2}}-\varepsilon_{3}^{\prime} \vartheta_{\mu_{3}}\right) \\
& \times\left(\vartheta_{\mu_{0}}-\varepsilon_{1}^{\prime} \vartheta_{\mu_{1}}+\varepsilon_{2}^{\prime} \vartheta_{\mu_{2}}-\varepsilon_{3}^{\prime} \vartheta_{\mu_{3}}\right)\left(\vartheta_{\mu_{0}}-\varepsilon_{1}^{\prime} \vartheta_{\mu_{1}}-\varepsilon_{2}^{\prime} \vartheta_{\mu_{2}}+\varepsilon_{3}^{\prime} \vartheta_{\mu_{3}}\right),
\end{aligned}
$$

\begin{tabular}{|c|c|c|c|c|}
\hline$\#$ & $\sigma \in G_{5} \backslash G$ & $x_{J_{5}}^{\sigma}$ & $\varepsilon, \varepsilon_{0} ; \varepsilon_{1}^{\prime}, \varepsilon_{2}^{\prime}, \varepsilon_{3}^{\prime}$ & $\begin{array}{l}\mu_{0} \\
\mu_{1} \\
\mu_{2} \\
\mu_{3}\end{array}$ \\
\hline 58 & id & $x_{\langle 13 ; 25 ; 47 ; 68\rangle}$ &,$+ 1 ; 1,1,1$ & 111111,000000 \\
\hline 59 & $g_{3}$ & $x_{\langle 14 ; 25 ; 37 ; 68\rangle}$ &,$+ 1 ; i, i, 1$ & 110000,001111 \\
\hline 60 & $g_{5}$ & $x_{\langle 13 ; 26 ; 47 ; 58\rangle}$ &,$+ 1 ; 1, i, i$ & 001100, 110011 \\
\hline 61 & $g_{3} g_{5}$ & $x_{\langle 14 ; 26 ; 37 ; 58\rangle}$ &,$+ 1 ; i,-1, i$ & 000011,111100 \\
\hline 62 & $g_{1}$ & $x_{\langle 23 ; 15 ; 47 ; 68\rangle}$ &,$+ 1 ; 1,1,1$ & 011111,100000 \\
\hline 63 & $g_{1} g_{3}$ & $x_{\langle 24 ; 15 ; 37 ; 68\rangle}$ &,$+ 1 ; i, i, 1$ & 010000,101111 \\
\hline 64 & $\overline{g_{1} g_{5}}$ & $x_{\langle 23 ; 16 ; 47 ; 58\rangle}$ &,$+ 1 ; 1, i, i$ & 101100, 010011 \\
\hline 65 & $g_{1} g_{3} g_{5}$ & $x_{\langle 24 ; 16 ; 37 ; 58\rangle}$ &,$+ 1 ; i,-1, i$ & 100011,011100 \\
\hline 66 & $g_{7}$ & $x_{\langle 13 ; 25 ; 48 ; 67\rangle}$ &,$+ 1 ; 1,1,1$ & 111110,000001 \\
\hline 67 & $g_{3} g_{7}$ & $x_{\langle 14 ; 25 ; 38 ; 67\rangle}$ &,$+ 1 ; i, i, 1$ & 110001,001110 \\
\hline 68 & $g_{5} g_{7}$ & $x_{\langle 13 ; 26 ; 48 ; 57\rangle}$ &,$+ 1 ; 1, i, i$ & 001101, 110010 \\
\hline 69 & $g_{3} g_{5} g_{7}$ & $x_{\langle 14 ; 26 ; 38 ; 57\rangle}$ &,$+ 1 ; i,-1, i$ & 000010,111101 \\
\hline 70 & $g_{1} g_{7}$ & $x_{\langle 23 ; 15 ; 48 ; 67\rangle}$ &,$+ 1 ; 1,1,1$ & 011110,100001 \\
\hline 71 & $g_{1} g_{3} g_{7}$ & $x_{\langle 24 ; 15 ; 38 ; 67\rangle}$ &,$+ 1 ; i, i, 1$ & 010001, 101110 \\
\hline 72 & $g_{1} g_{5} g_{7}$ & $x_{\langle 23 ; 16 ; 48 ; 57\rangle}$ &,$+ 1 ; 1, i, i$ & 101101, 010010 \\
\hline 73 & $g_{1} g_{3} g_{5} g_{7}$ & $x_{\langle 24 ; 16 ; 38 ; 57\rangle}$ &,$+ 1 ; i,-1, i$ & 100010,011101 \\
\hline
\end{tabular}

where $\varepsilon=\varepsilon_{0}^{4}, \varepsilon_{0}, \varepsilon_{j}^{\prime}=\varepsilon_{j} / \varepsilon_{0}$ and $\mu_{0}, \ldots, \mu_{3}$ are listed in the following table. 


\begin{tabular}{|c|c|c|c|c|}
\hline 74 & $h_{34}$ & $-x_{\langle 13 ; 27 ; 45 ; 68\rangle}$ &,$- \zeta ; 1, i, i$ & 110110,000110 \\
\hline 75 & $g_{3} h_{34}$ & $-x_{\langle 14 ; 27 ; 35 ; 68\rangle}$ &,$- \zeta ; i,-1, i$ & 111010,001010 \\
\hline 76 & $g_{5} h_{34}$ & $-x_{\langle 13 ; 28 ; 45 ; 67\rangle}$ &,$- \zeta ; 1,-1,-1$ & 000101,110101 \\
\hline 77 & $g_{3} g_{5} h_{34}$ & $-x_{\langle 14 ; 28 ; 35 ; 67\rangle}$ &,$- \zeta ; i,-i,-1$ & 001001,111001 \\
\hline 78 & $g_{1} h_{34}$ & $-x_{\langle 23 ; 17 ; 45 ; 68\rangle}$ &,$- \zeta ; 1, i, i$ & 010110,100110 \\
\hline 79 & $g_{1} g_{3} h_{34}$ & $-x_{\langle 24 ; 17 ; 35 ; 68\rangle}$ &,$- \zeta ; i,-1, i$ & 011010,101010 \\
\hline 80 & $g_{1} g_{5} h_{34}$ & $-x_{<}$ &,$- \zeta ; 1,-1,-1$ & 100101, 010101 \\
\hline 81 & $g_{1} g_{3} g_{5} h_{34}$ & $-x_{\langle 24 ;}$ &,$- \zeta ; i,-i,-1$ & 101001, 011001 \\
\hline 82 & $g_{7} h_{34}$ & $-x_{\langle 13 ; 27 ; 46 ; 58\rangle}$ &,$- \zeta ; 1,-i, i$ & 111100,000000 \\
\hline 83 & $g_{3} g_{7} h_{34}$ & $-x_{\langle 1}$ &,$- \zeta ; i, 1, i$ & 110000,001100 \\
\hline 84 & $g_{5} g_{7} h_{34}$ & $-x_{\langle 13 ; 28 ; 46 ; 57\rangle}$ &,$- \zeta ; 1,1,-1$ & 001111,110011 \\
\hline 85 & $g_{3} g_{5} g_{7} h_{34}$ & $-x_{\langle 14 ; 28 ; 36 ; 57\rangle}$ &,$- \zeta ; i, i,-1$ & 000011,111111 \\
\hline 86 & $g_{1} g_{7} h_{34}$ & $-x_{\langle 23}$ &,$- \zeta ; 1,-i, i$ & 011100,100000 \\
\hline 87 & $g_{1} g_{3} g_{7} h_{34}$ & $-x_{\langle 24 ; 17 ; 36 ; 58\rangle}$ &,$- \zeta ; i, 1, i$ & 010000,101100 \\
\hline 88 & $g_{1} g_{5} g_{7} h_{34}$ & $-x_{\langle 23 ; 18 ; 46 ; 57\rangle}$ &,$- \zeta ; 1,1,-1$ & 101111, 010011 \\
\hline 89 & $g_{1} g_{3} g_{5} g_{7} h_{34}$ & $-x_{\langle 2}$ &,$- \zeta ; i, i,-1$ & 100011,011111 \\
\hline 90 & $h_{24}$ & $x_{\langle 17}$ &,$+ 1 ; i,-1,-i$ & 111000,000100 \\
\hline 91 & $g_{3} h_{24}$ & $x_{\langle 1 \varepsilon}$ &,$+ 1 ;-1,-i,-$ & 110111, 001011 \\
\hline 92 & $g_{5} h_{24}$ & $x_{\langle 17 ; 2}$ &,$+ 1 ; i,-i, 1$ & 001011,110111 \\
\hline 93 & $g_{3} g_{5} h_{24}$ & $x_{\langle 1}$ &,$+ 1 ;-1,1,1$ & 000100,111000 \\
\hline 94 & $g_{1} h_{24}$ & $x_{\langle 27 ;}$ &,$+ 1 ; i,-1,-i$ & 011000,100100 \\
\hline 95 & $g_{1} g_{3} h_{24}$ & $x_{\langle 28 ; 15 ; 37 ; 46\rangle}$ &,$+ 1 ;-1,-i,-i$ & 010111, 101011 \\
\hline 96 & $g_{1} g_{5} h_{24}$ & $x_{\langle 2}$ &,$+ 1 ; i,-i, 1$ & 101011, 010111 \\
\hline 97 & $g_{1} g_{3} g_{5} h_{24}$ & $x_{\langle 28 ; 16 ; 37 ; 45\rangle}$ &,$+ 1 ;-1,1,1$ & 100100,011000 \\
\hline 98 & $g_{7} h_{24}$ & $x_{\langle 17 ; 25 ; 48 ; 36\rangle}$ &,$+ 1 ; i,-1, i$ & 100000,010000 \\
\hline 99 & $g_{3} g_{7} h_{24}$ & $x_{\langle 1}$ &,$+ 1 ; 1,-i, i$ & 101111,011111 \\
\hline 100 & $g_{5} g_{7} h_{24}$ & $x_{\langle 17 ; 26 ; 48 ; 35\rangle}$ &,$+ 1 ;,-i,-1$ & 010011,100011 \\
\hline 101 & $g_{3} g_{5} g_{7} h_{24}$ & $x_{\langle 18 ; 26 ; 47 ; 35\rangle}$ &,$+ 1 ;-1,1,-1$ & 011100,101100 \\
\hline 102 & $g_{1} g_{7} h_{24}$ & $x_{\langle 2}$ &,$+ 1 ; i,-1, i$ & 000000,110000 \\
\hline 103 & $g_{1} g_{3} g_{7} h_{24}$ & $x_{\langle 28 ; 15 ; 47 ; 36\rangle}$ &,$+ 1 ;-1,-i, i$ & 001111,111111 \\
\hline 104 & $g_{1} g_{5} g_{7} h_{24}$ & $x_{\langle 27 ; 1 ; 48 ; 35\rangle}$ &,$+ 1 ; i,-i,-1$ & 110011,000011 \\
\hline 105 & $g_{1} g_{3} g_{5} g_{7} h_{24}$ & $x_{\langle 2}$ &,$+ 1 ;-1,1,-1$ & 111100,001100 \\
\hline
\end{tabular}




\section{Some relations among $\vartheta_{a, b}(y)$ on $\mathbb{B}^{5}$}

\subsection{Quadratic relations among $\vartheta_{a, b}(y)$ on $\mathbb{B}^{5}$}

Proposition 8 The functions $\vartheta_{a, b}(y)$ on $\mathbb{B}^{5}$ satisfy the following quadratic relations:

(1) For $\mu_{0}, \ldots, \mu_{3}$ and $\varepsilon$ in Theorem 1 ,

$$
\vartheta_{\mu_{0}}(y) \vartheta_{\mu_{2}}(y)=\varepsilon \vartheta_{\mu_{1}}(y) \vartheta_{\mu_{3}}(y)
$$

(2) For $\mu_{0}, \ldots, \mu_{3}$ and $\varepsilon_{0}, \ldots, \varepsilon_{3}$ in Theorem 2,

$$
\varepsilon_{0} \varepsilon_{3} \vartheta_{\mu_{0}}(y) \vartheta_{\mu_{3}}(y)=-\varepsilon_{1} \varepsilon_{2} \vartheta_{\mu_{1}}(y) \vartheta_{\mu_{2}}(y) ;
$$

(3) For $\mu_{0}, \ldots, \mu_{3}$ and $\varepsilon^{\prime}$ in Theorem 3,

$$
\vartheta_{\mu_{0}}^{2}(y)+\varepsilon^{\prime} \vartheta_{\mu_{1}}^{2}(y)=\vartheta_{\mu_{2}}^{2}(y)+\varepsilon^{\prime} \vartheta_{\mu_{3}}^{2}(y)
$$

(4) For $\mu_{0}, \ldots, \mu_{3}$ and $\varepsilon_{1}^{\prime}, \varepsilon_{2}^{\prime}, \varepsilon_{3}^{\prime}$ in Theorem 4 ,

$$
\vartheta_{\mu_{0}}(y) \vartheta_{\mu_{2}}(y)=\varepsilon_{1}^{\prime} \varepsilon_{2}^{\prime-1} \varepsilon_{3}^{\prime} \vartheta_{\mu_{1}}(y) \vartheta_{\mu_{3}}(y) .
$$

Proof. (1) Act $g_{2}$ on the equality $\vartheta_{m_{0}}(y) \vartheta_{m_{2}}(y)=\vartheta_{m_{1}}(y) \vartheta_{m_{3}}(y)$ in Fact 4 (2). Then we have

$$
\vartheta_{\mu_{0}}(y) \vartheta_{\mu_{2}}(y)=\vartheta_{\mu_{1}}(y) \vartheta_{\mu_{3}}(y)
$$

for $\mu_{j}$ in Proposition 4. Act $\sigma \in G_{2} \backslash G$ on this equality.

(2) Act $g_{4}$ on the equality $\vartheta_{\mu_{0}}(y) \vartheta_{\mu_{2}}(y)=\vartheta_{\mu_{1}}(y) \vartheta_{\mu_{3}}(y)$ for $\mu_{j}$ in \#2 of the table in Theorem 1 . Then we have

$$
\begin{aligned}
\left(\vartheta_{\nu_{0}}(y)+\vartheta_{\nu_{1}}(y)\right)\left(\vartheta_{\nu_{2}}(y)+\right. & \left.\vartheta_{\nu_{3}}(y)\right) \\
& =\left(\vartheta_{\nu_{0}}(y)-\vartheta_{\nu_{1}}(y)\right)\left(\vartheta_{\nu_{2}}(y)-\vartheta_{\nu_{3}}(y)\right),
\end{aligned}
$$

where $\nu_{j}$ are in \#14 of the table in Theorem 2. This equality is equivalent to $\vartheta_{\nu_{0}}(y) \vartheta_{\nu_{3}}(y)=-\vartheta_{\nu_{1}}(y) \vartheta_{\nu_{2}}(y)$. Act $\sigma \in G_{3} \backslash G$ on this equality.

(3) Act $g_{6}$ on the equality $\vartheta_{\mu_{0}}(y) \vartheta_{\mu_{2}}(y)=\vartheta_{\mu_{1}}(y) \vartheta_{\mu_{3}}(y)$ for $\mu_{j}$ in \#2 of the table in Theorem 1 . Then we have

$$
\vartheta_{\nu_{0}}^{2}(y)-\vartheta_{\nu_{1}}^{2}(y)=\vartheta_{\nu_{2}}^{2}(y)-\vartheta_{\nu_{3}}^{2}(y)
$$

where $\nu_{j}$ are in \#46 of the table in Theorem 3. Act $\sigma \in G_{4} \backslash G$ on this equality. 
(4) Act $g_{4}$ on the equality

$$
\vartheta_{\mu_{0}}^{2}(y)-\vartheta_{\mu_{1}}^{2}(y)=\vartheta_{\mu_{2}}^{2}(y)-\vartheta_{\mu_{3}}^{2}(y),
$$

for $\mu_{j}$ in \#46 of the table in Theorem 3 . Then we have

$$
\begin{aligned}
&\left(\vartheta_{\nu_{0}}(y)+\vartheta_{\nu_{1}}(y)+\vartheta_{\nu_{2}}(y)+\vartheta_{\nu_{3}}(y)\right) \times\left(\vartheta_{\nu_{0}}(y)-\vartheta_{\nu_{1}}(y)+\vartheta_{\nu_{2}}(y)-\vartheta_{\nu_{3}}(y)\right) \\
&=\left(\vartheta_{\nu_{0}}(y)+\vartheta_{\nu_{1}}(y)-\vartheta_{\nu_{2}}(y)-\vartheta_{\nu_{3}}(y)\right) \\
& \times\left(\vartheta_{\nu_{0}}(y)-\vartheta_{\nu_{1}}(y)-\vartheta_{\nu_{2}}(y)+\vartheta_{\nu_{3}}(y)\right),
\end{aligned}
$$

where $\nu_{j}$ are in \#58 of the table in Theorem 4. This equality is equivalent to $\vartheta_{\nu_{0}}(y) \vartheta_{\nu_{2}}(y)=\vartheta_{\nu_{1}}(y) \vartheta_{\nu_{3}}(y)$. Act $\sigma \in G_{5} \backslash G$ on this equality.

\subsection{Relations among $f_{J}$ corresponding to those among $x_{J}$}

The polynomials $x_{J}$ satisfy the relations

$$
x_{\left\langle j_{1} j_{2} ; j_{3} j_{4} ; j_{5} j_{6} ; j_{7} j_{8}\right\rangle}-x_{\left\langle j_{1} j_{3} ; j_{2} j_{4} ; j_{5} j_{6} ; j_{7} j_{8}\right\rangle}+x_{\left\langle j_{1} j_{4} ; j_{2} j_{3} ; j_{5} j_{6} ; j_{7} j_{8}\right\rangle}=0,
$$

where $j_{1}<j_{2}<j_{3}<j_{4}$. Thus $f_{J}(y)$ satisfy the same relations. For examples,

$$
f_{\langle 12 ; 34 ; 56 ; 78\rangle}(y)-f_{\langle 13 ; 24 ; 56 ; 78\rangle}(y)+f_{\langle 14 ; 23 ; 56 ; 78\rangle}(y)=0,
$$

and

$$
f_{\langle 25 ; 68 ; 14 ; 37\rangle}(y)-f_{\langle 26 ; 58 ; 14 ; 37\rangle}(y)+f_{\langle 28 ; 56 ; 14 ; 37\rangle}(y)=0 .
$$

By \#27, \#59 and \#61 in the tables in Theorems 1 and 4, the left hand side of the second equality becomes

$$
2\left(\vartheta_{\mu_{0}}(y) \vartheta_{\mu_{2}}(y)-\vartheta_{\mu_{1}}(y) \vartheta_{\mu_{3}}(y)\right)^{2},
$$

where

$$
\left(\begin{array}{l}
\mu_{0} \\
\mu_{1} \\
\mu_{2} \\
\mu_{3}
\end{array}\right)=\left(\begin{array}{l}
111111,000000 \\
110000,001111 \\
001100,110011 \\
000011,111100
\end{array}\right) .
$$

Note that

$$
\vartheta_{\mu_{0}}(y) \vartheta_{\mu_{2}}(y)-\vartheta_{\mu_{1}}(y) \vartheta_{\mu_{3}}(y)=0
$$

by Proposition 8 . 


\section{References}

[I] Igusa J., Theta Functions. Springer, 1972.

[K] Kondo S., The moduli space of 8 points on $\mathbf{P}^{1}$ and automorphic forms. Algebraic Geometry (ed. by J.H. Keum, S. Kondo), Contemporary Mathematics vol. 422, 89-106, A.M.S.

[MT] Matsumoto K. and Terasoma T., Theta constants associated to coverings of $\mathbf{P}^{1}$ branching at 8 points. Compositio Math. 140 (2004), 1277-1301.

[MY] Matsumoto K. and Yoshida M., Configuration space of 8 points on the projective line and a 5-dimensional Picard modular group. Compositio Math. 86 (1993), $265-280$

K. Matsumoto

Department of Mathematics

Hokkaido University

Sapporo, 060-0810 Japan

E-mail: matsu@math.sci.hokudai.ac.jp

T. Minowa

Tajimi-Kita High-School

Kamiyama 2-49, Tajimi

Gifu, 507-0022 Japan

R. Nishimura

Sapporo Public Employment Security Office Minami-10 Nishi-14, Chuo-ku

Sapporo, 064-8609 Japan 